CAE Working Paper \#04-13

On Choice of Technique in the Robinson-Solow-Srinivasan Model

by

M. Ali Khan

and

Tapan Mitra

July 2004 


\title{
On Choice of Technique in the Robinson-Solow-Srinivasan Model ${ }^{*}$
}

\author{
M. Ali Khan ${ }^{\dagger}$ and Tapan Mitra ${ }^{\ddagger}$
}

September 2003

\begin{abstract}
We report results on the optimal "choice of technique" in a model originally formulated by Robinson, Solow and Srinivasan. By viewing this model as a specific instance of the general theory of intertemporal resource allocation associated with Brock, Gale and McKenzie, we resolve long-standing conjectures in the form of theorems on the existence and price-support of optimal paths, and on their long-run behavior. We also examine policies, due to Stiglitz, as a cornerstone for a theory of transition dynamics in this model. We present examples to show that (i) an optimal program can be periodic, (ii) a Stiglitz' program can be bad, and (iii) a Stiglitz production program can be non-optimal. We then provide sufficient conditions under which the policies proposed by Stiglitz coincide with optimal behavior. (127 words)
\end{abstract}

Journal of Economic Literature Classification Numbers: D90, C62, O21.

Key Words: Choice of technique, overtaking criterion, optimal program, golden-rule stock, golden-rule price, value-loss, average turnpike property, long-run, transition dynamics, Stiglitz program, Stiglitz production program, price-support property, cycling.

Running Title: Choice of Technique

${ }^{*}$ The work reported here is part of a project with a long gestation period: it was initiated during Mitra's visit to the Department of Economics at the University of Illinois in 1986, received invaluable impetus from Professor Robert Solow's presentation at the Srinivasan Conference held at Yale in March 1998, was continued when Khan visited the Department of Economics at Cornell in November 1998, October 2000, July 2003 and July 2004, and the EPGE, Fundação Getilio Vargas in January 2001 and December 2002. The authors are grateful to all of these institutions for their hospitality, as well as to the Center for Analytic Economics at Cornell and the Center for a Livable Future at Johns Hopkins for research support. Two anonymous referees of IJET and Mr. Chris Metcalf gave the manuscript a careful reading. Khan also thanks Professors Abhijit Banerjee, Jimmy Chan, Avinash Dixit and Debraj Ray for stimulating conversation.

$\dagger$ Department of Economics, The Johns Hopkins University, Baltimore, MD 21218.

$\ddagger$ Department of Economics, Cornell University, Ithaca, New York 14853 . 


\section{Introduction}

In the late sixties and early seventies, under the general heading of "technical choice under full employment in a socialist economy," Robinson (1960, 1969), Okishio (1966) and Stiglitz (1968, 1970, 1973) studied the problem of optimal economic growth in a model of an economy originally formulated by Robinson (1960, pp. 38-56), Solow (1962b) and Srinivasan (1962a) (henceforth, the RSS model). ${ }^{1}$ The work generated controversy. Stiglitz argued, with justification, that the Robinson-Okishio assumption of a fixed labor allocation between the consumption and investment sectors had no place in an exercise that sought to determine the optimal growth path, and thereby an optimum time-path of the allocation of labor. ${ }^{2}$ He identified development policies, henceforth Stiglitz' policies, ${ }^{3}$ under which there is investment only in the type of machine $\sigma$ that minimizes effective labor costs and simultaneously maximizes the steady state consumption, and a utilization of only those types of machines whose output per man ratios are higher than the effective labor cost of producing $\sigma$. Stiglitz observed that the "number of workers working in the consumption-goods sector increases monotonically (capital 'widening' occurs in a smooth way), output of consumption goods need not be monotonically increasing", ${ }^{4}$ and prescribed for the economy at any point in time an optimal choice of techniques, both to use and to produce, and thereby the (instantaneous) optimal levels of technological obsolescence - prescriptions which are all independent of the felicity function. Robinson commented on Stiglitz' solution by criticizing his assumption of a fixed positive discount rate, continuous time and the linearity assumption in the specification of the planner's felicity function. ${ }^{5}$

Robinson's objections were explicitly acknowledged by Stiglitz, ${ }^{6}$ and as a first approximate step, ${ }^{7}$ he extended his earlier analysis to the case of a minimum consumption constraint in a setting with continuous time and a positive rate of discount. However, he emphasized that the important modifications concerned transition, rather than long-run, dynamics. ${ }^{8}$

Even if there is a minimum consumption constraint and a finite gestation period, the path of development will, after an initial "adjustment" period, look exactly as I have described it. [Unlike] long-run neoclassical models with malleable capital [where] the optimal policy

\footnotetext{
${ }^{1}$ In Khan (2000, p.3), the model is referred to as the Solow-Srinivasan model; also see Solow (1962, concluding three paragraphs) and Khan (2000, Footnote 12) for the way it is seen in earlier work.

${ }^{2}$ See $(1968$, Paragraph $1 ; 1970$, p. 421). Stiglitz (1970, p. 421) writes "There may be some special situations ... where the employment allocation is the same for all steady-state paths, but even then, in going from one steady-state path to another, one cannot infer that the employment allocation is unchanged - and it is this dynamic problem that we are discussing."

${ }^{3}$ This is formalized in Definitions 8 and 9 below. As we shall see in the sequel, Stiglitz' policies can be usefully compared to Faustmann's solution to the forestry problem, as formalized in Mitra-Wan (1986).

${ }^{4}$ See Stiglitz (1973, pp. 143-144). In the discussion of his policy, Stiglitz also drew attention to preliminary investigations of Bruno (1967).

${ }^{5}$ We restate Robinson's criticisms in our own terminology; she phrases them in terms of a "discount rate chosen once and for all, ... negligible gestation periods, ...[and] ceasing to consume and living on air during the first phase of the plan."

${ }^{6}$ See Stiglitz (1973, p. 144) and also Cass-Stiglitz (1969, p. 614).

${ }^{7}$ Thus Cass-Stiglitz (1969, Footnote 19) saw the instantaneous utility function $U(C)=-\infty$ for $C<\bar{C}$ and $U(C)=C$ for $C \geq \bar{C}, \bar{C}$ a minimum consumption level, as "one approximation to the general instantaneous utility function satisfying $U^{\prime}(C)>0$ with $\lim _{C=0} U^{\prime}(C)=\infty$ and $U^{\prime \prime}(C) \leq 0$."

${ }^{8}$ Stiglitz's (1970) response is important for the record; this essay can also be seen as a further investigation into the substance of this response.
} 
is always of the so-called bang-bang variety - if the initial capital labor ratio is less than its long-run equilibrium value there is always a period of zero consumption, after which consumption jumps to its long-run equilibrium value, whereas in our ex-post fixed coefficients model consumption increases steadily to its long-run value.

Given the primary interest in the undiscounted case, Stiglitz interpreted the undiscounted case as a situation when the discount rate is "negligible"; he developed the intuitive ideas in discrete-time and then chose to translate them to the continuous-time framework. ${ }^{9}$

In his recent revisit of Srinivasan (1962a), Solow (2000, p. 7) asks for a solution to the "Ramsey problem for this model." Since Stiglitz had already provided a solution with a "linear utility and positive time preference", the open questions concern a rigorous treatment of the undiscounted case and of the discounted case with a "strictly concave social utility function for current per capita consumption." Like Robinson, Solow also mentions that an "adoption of this [linear utility] criterion can indeed lead to unjustifiable neglect of early consumption," and if one was to share "Ramsey's belief that the only ethically defensible social rate of time preference is zero, a sufficiently sharply-concave utility function would enforce a closer approach to intergenerational equality." In short, Solow's question remains unanswered, and the generalization of Stiglitz's work in the directions it prompts remains yet to be accomplished. ${ }^{10}$ In this paper, we address this general question, and most importantly from a methodological point of view, do so in the setting of the modern theory of optimal intertemporal allocation initiated originally by Ramsey and von Neumann and brought to completion at the hands of Brock, Gale and McKenzie. ${ }^{11}$ Since this theory was being finalized at the same time as the "capital controversy" between the two Cambridges, ${ }^{12}$ it has not been brought to bear on the fundamental issues.

In terms of specifics, we truly treat the Ramsey problem; that is, consider a formulation in which there is no discounting of future utilities, and thus no appeal to the assumption of structural stability of the model at the zero discount rate, an assumption at best roundabout and at worst dubious. We are by this time very familiar with the overtaking criterion of Atsumi (1965) and von Weiszäcker (1965), and under this criterion, an optimal path in the undiscounted case can be shown to exist and its properties can be rigorously studied. Our treatment of time is discrete: the general theory of intertemporal allocation is developed in the simplest and most elegant way in such a setting (see McKenzie (1986) for a masterly presentation), and we can work with a reduced form of the RSS model ${ }^{13}$ in which the technological possibilities are given by a transition possibility set, and the objective function by

\footnotetext{
${ }^{9}$ See Footnotes 1 and 3 on page 608 and the discussion of the "correct" pricing system on page 606 in Stiglitz (1968).

${ }^{10}$ For some partial attempts at solution, and for a numerical example, see Stiglitz (1973); also see Stiglitz (1968, Footnote 2, p. 608) and Cass-Stiglitz (1969). However, in Khan (2000, p. 15), the situation is expressed as "The loose end remains loose."

${ }^{11}$ The relevant papers are Gale (1967), McKenzie (1968) and Brock (1970). In the sequel, when we refer to the "general theory of optimal intertemporal allocation", we shall be having these papers in mind.

${ }^{12}$ It is of course not our intention to revisit this debate here - the interested reader may want to see Birner (2002) and his references.

${ }^{13}$ We choose to work with the version presented in Stiglitz (1968) rather than that in Solow (1962b) or Srinivasan (1962a). All of these variants can be viewed as special cases of the models considered in Bruno (1967) or the more general treatment in Koopmans (1971) and Koopmans-Hansen (1972). We leave the analysis of these papers as a task for future research; also see the third paragraph of the concluding Section 8 below.
} 
a (reduced-form) utility function defined on this set (that is, defined on beginning and end of period capital stock vectors). We establish the existence of a golden-rule stock, with support prices, and show that the golden-rule stock is unique. We appeal to the methods of Brock (1970) and McKenzie (1968) to show the existence of an optimal program, and furthermore, to establish that, starting from an arbitrary initial stock, it converges asymptotically to a subset of the transition set, the so-called von Neumann facet, consisting of all plans which have "zero value-loss" at the golden-rule support prices. In the case of a strictly concave felicity function, the von Neumann facet shrinks to a point, and so we have asymptotic convergence to the golden-rule stock. These results furnish a complete resolution of the problem of the long-run choice of technique and thereby illustrate the power and elegance of the general theory.

Since we have a complete resolution of the problem of the long-run choice of technique, the natural question arises as to the choice of technique in transition to the steady state: a determination of the type and amounts of machines that are produced and used in the short run. Unfortunately, it is on this hard problem of transition dynamics that the general theory has little to offer, with the literature lacking concrete results of any generality. ${ }^{14}$ However, Stiglitz' prescriptions as to the choice of techniques can be identified as a basis for the development of a full-scale theory of transition dynamics - an analytical marker at which one can aim. It is here that the results yield surprises: even questions once seen as resolved are now starkly revealed not to be so through simple and compelling counterexamples.

Moving on to positive results, for an economy with a linear felicity function, we offer a (novel) set of sufficient conditions, pertaining only to the parameters of the type of machine $\sigma$ used in the long-run, under which the Stiglitz program is optimal, and uniquely so. For economies with a general felicity function, and a fortiori, for an economy with a linear utility function and a minimum consumption constraint, we also present sufficient conditions for the optimality of a Stiglitz production program. These conditions, in pointing to an interesting distinction between choice of technique that is appropriate in the short-run from that which is appropriate in the long-run, also connects to the literature of the sixties on planning in India (and elsewhere) ${ }^{15}$ that comes as close to stating the problem as precisely as can be expected in the pre-Pontryagin period. ${ }^{16}$ However, this literature and earlier work notwithstanding, it will generally be recognized today that whether we are interested in this issue from a planning perspective or from the modern perspective of a representative agent, the problem of an appropriate choice of technique should really be viewed as part of the general theory of economic growth. A subsidiary motivation of this paper is to facilitate this re-orientation. ${ }^{17}$

It is important to appreciate the methodological significance of this reformulation of the RSS model. In the standard treatment based on Pontryagin's principle, ${ }^{18}$ as in the work of Stiglitz (1968), Sen

\footnotetext{
${ }^{14}$ See the third paragraph of the concluding remarks in Section 8 below.

${ }^{15}$ In addition to Raj-Sen (1961) and Sen (1960) in particular, also see Dobb (1956, 1960, 1961, 1967), Halevi (1987), Mirrlees (1962), Naqvi (1963), Solow (1962a); and for an open-economy perspective, Bardhan (1971).

${ }^{16}$ See Raj-Sen (1961, pp. 48, 51 and concluding section). We remind the reader that an earlier version of the Raj-Sen paper (with a different title) was published in Arthaniti in 1959, and that Naqvi (1963) is a follow-up to the Raj-Sen paper as his leading footnote clearly indicates.

${ }^{17}$ For an early emphasis on this, see Mirrlees (1962) and Srinivasan (1962b); Okishio (1987) represents the alternative perspective. This point was independently underscored to Khan by Debraj Ray as a comment on Khan (2000).

${ }^{18}$ Thus Dixit (1990, p. 5) writes, "Nowadays Hamiltonians and phase diagrams are everyday stuff for the typical second-
} 
(1968) and others, one appeals to the transversality conditions in the study of the differential equations pertaining to the state and auxiliary variables obtained by substituting the values of the controls that maximize the instantaneous Hamiltonian. ${ }^{19}$ Thus the relevance of the rest points is established only towards the end of the analysis. Here, we begin with the rest points, the golden-rule stock and the golden-rule prices, and use the value-loss function and the so-called average turnpike property of good programs to yield the optimal program. ${ }^{20}$ Stiglitz investigates the convergence (turnpike property) of a path that follows his (derived) policy prescriptions as to the choice of techniques, ${ }^{21}$ while we need to investigate whether the optimal path and its turnpike property is sustained by these prescriptions. As mentioned above, this cannot be established, in general, for either a linear or a strictly concave felicity function, but only in special (identified) cases of either formulation. Thus, through the introduction of a new conceptual vocabulary, a difficult step in one perspective is rendered straightforward in another. ${ }^{22}$

In sum, our results exhibit in a dramatic way both the strength and the weakness of the general theory of intertemporal allocation alluded to earlier, and thereby reveal exactly why the choice of an appropriate technique is such a difficult and multi-facetted problem. The application has the advantage of illustrating the power and flexibility of the modern theory: it is ideally suited to deal with this problem, and the general results of this theory can be readily applied through the use of extremely elementary methods. As such it is perhaps overdue. However, a secondary benefit of this application concerns the theory itself; it offers insights into its scope and suggests directions along which it may find fruitful extension. ${ }^{23}$ It also points clearly to issues to which the general theory has (and by its very nature, will have) very little to offer, thereby indicating that even after much theoretical progress has been made, some of the questions that were asked fifty years ago about the appropriate choice of technique remain hard unanswered problems that need to be approached case by case.

The remainder of the paper is organized as follows. Sections 2, 3 and 4 present the basic theory of the RSS model when it is converted to its Gale-McKenzie reduced form. In particular, under a standing hypothesis on the finite set of parameters that define the RSS model, we show the existence and uniqueness of the golden-rule stock, and the existence of a program that is optimal starting from any given initial stock of machines. With this standard theory (Theorems 1 to 2 ) as an (indispensable) background, we can turn to the central results of the paper. In Section 5 , we consider the question of the

year graduate student", and quotes Frank Hahn's reference to the "unseemly haste to get down to the Hamiltonian."

${ }^{19}$ In the context of Stiglitz (1968), see his Footnote 2 both on page 605 and on page 607.

${ }^{20}$ As the reader will see below, we appeal to McKenzie's $(1983,1986)$ price-support property only to establish our final result pertaining to transition dynamics in the case of a strictly concave felicity function (Theorem 7 below).

${ }^{21}$ As alluded to in Footnote 3, we see the Stiglitz policy as the analogue of the Faustmann solution in the economics of forestry, and it would be interesting to pursue the analytics of this analogy; see the fourth paragraph of the concluding remarks in Section 8 below.

${ }^{22}$ Stiglitz (1968, Footnote 2, p. 608) recognizes that the results for the linear utility function might not carry over to the general concave case, and in a subsequent analysis of the problem, one with a minimum consumption constraint, he refers to the difficulty of showing that there is only one type of machine that maximizes $p(x, t)$, see Stiglitz (1973; p. 145). He also refers in this connection to Bliss (1968) and Cass-Stiglitz (1969). For this scepticism concerning the linear case, also see Solow (2000) in addition to Robinson (1969).

${ }^{23}$ Thus, a distinction has to be drawn between applying a theorem from applying its methods of proof. As the reader will see in the sequel, the hypotheses of Brock's existence theorem and of McKenzie's price-support property are not literally fulfilled by the RSS model but their methods of proof $d o$ apply. 
correct choice of technique for the long-run, and through the identification of the von Neumann facet, present results for both linear and strictly concave felicity functions. In Section 6, we turn to transition dynamics through the identification and formalization of the policy prescriptions due to Stiglitz (1968), and present examples that decisively refute plausible intuitions concerning these prescriptions. In Section 7, we present a sufficient parameterization under which a Stiglitz program and/or a Stiglitz production program is optimal. The concluding Section 8 lists the salient results and identifies problems that remain open. The technical and computational details of the proofs are collected in an Appendix.

\section{The Model and its Reduced Form}

We begin with some preliminary notation. Let $\mathbb{N}\left(\mathbb{N}_{+}\right)$be the set of non-negative (positive) integers, $\mathbb{R}\left(\mathbb{R}_{+}\right)$the set of real (non-negative) numbers. We shall work in finite-dimensional Euclidean space $\mathbb{R}^{n}$ with non-negative orthant $\mathbb{R}_{+}^{n}=\left\{x \in \mathbb{R}^{n}: x_{i} \geq 0, i=1, \cdots, n\right\}$. For any $x, y$ in $\mathbb{R}^{n}$, let the inner product $x y=\sum_{i=1}^{n} x_{i} y_{i}$, and $x>>y, x>y, x \geq y$ have their usual meaning. Let $e(i), i=1, \cdots, n$, be the $i^{t h}$ unit vector in $\mathbb{R}^{n}$, and $e$ be an element of $\mathbb{R}_{+}^{n}$ all of whose coordinates are unity. For any $x \in \mathbb{R}^{n}$, let $\|x\|$ denote the Euclidean norm of $x$. The empty set is denoted by $\emptyset$ and set-theoretic subtraction between $A$ and $B$ by $A / B$.

Our choice of $\mathbb{R}^{n}$ is dictated by the consideration of an economy capable of producing a finite number $n$ of alternative types of machines. For every $i=1, \cdots, n$, one unit of machine of type $i$ requires $a_{i}>0$ units of labor to construct it, and together with one unit of labor, each unit of it can produce $b_{i}>0$ units of a single consumption good. Thus, the production possibilities of the economy can be represented by an (labor) input-coefficients vector, $a=\left(a_{1}, \cdots, a_{n}\right)>>0$ and an output-coefficients vector, $b=\left(b_{1}, \ldots, b_{n}\right)>>0$. Without loss of generality ${ }^{24}$ we shall assume that the types of machines are numbered such that $b_{1} \geq b_{2} \cdots \geq b_{n}$.

We shall assume that all machines depreciate at a rate $d \in(0,1)$. Thus the effective labor cost of producing a unit of output on a machine of type $i$ is given by $\left(1+d a_{i}\right) / b_{i}$ : the direct labor cost of producing unit output, and the indirect cost of replacing the depreciation of the machine in this production. ${ }^{25}$ We shall work with the reciprocal of the effective labor cost, the effective output that takes the depreciation into account, and $\operatorname{denote}^{26}$ it by $c_{i}$ for the machine of type $i$. Throughout this paper, we shall assume that there is a unique machine type $\sigma$ at which this effective labor cost $\left(1+d a_{i}\right) / b_{i}$ is minimized, or at which the effective output per man $b_{i} /\left(1+d a_{i}\right)$ is maximized. Thus, we shall assume:

$$
\text { There exists } \sigma \in\{1, \cdots, n\} \text { such that for all } i=1, \cdots, n, i \neq \sigma, c_{\sigma}>c_{i} \text {. }
$$

For each date $t \in \mathbb{N}$, let $x(t)=\left(x_{1}(t), \cdots, x_{n}(t)\right) \geq 0$ denote the amounts of the $n$ types of machines that are available in time-period $t$, and let $z(t+1)=\left(z_{1}(t+1), \cdots, z_{n}(t+1)\right) \geq 0$ be the gross

\footnotetext{
${ }^{24}$ Note that Stiglitz (1968) assumes that $b_{i}>b_{j}$ implies that $a_{i}>a_{j}$; whereas this is a natural hypothesis, we make no such assumption.

${ }^{25}$ See Stiglitz (1968, pp. 608-609) on a "labor theory of value" interpretation.

${ }^{26}$ As we shall see below, $c_{i}$ is the value of the steady-state consumption per man if only machines of type $i$ are used and produced, a consideration that governs our choice of notation.
} 
investments in the $n$ types of machines during period $(t+1)$. Hence, $z(t+1)=(x(t+1)-x(t))+d x(t)$, the sum of net investment and of depreciation. Let $y(t)=\left(y_{1}(t), \ldots, y_{n}(t)\right)$ be the amounts of the $n$ types of machines used for production of the consumption good, by $(t)$, during period $(t+1) .{ }^{27}$ Let the total labor force of the economy be stationary and positive. We shall normalize it to be unity. Clearly, gross investment, $z(t+1)$ representing the production of new machines of the various types, will require $a z(t+1)$ units of labor in period $t$. Also, $y(t)$ representing the use of available machines for manufacture of the consumption good, will require ey(t) units of labor in period $t$. Thus, the availability of labor constrains employment in the consumption and investment sectors by $a z(t+1)+e y(t) \leq 1$. Note that both the flow of consumption and of investment (new machines) are in gestation during the period and available at the end of it. We now give a formal summary of this technological structure.

Definition 1 A program from $x_{o}$ in $\mathbb{R}_{+}^{n}$ is a sequence ${ }^{28}\{x(t), y(t)\}$ with $(x(t), y(t)) \in \mathbb{R}_{+}^{n} \times \mathbb{R}_{+}^{n}$ such that $x(0)=x_{o}$, and for all $t \in \mathbb{N},(i) x(t+1) \geq(1-d) x(t),($ ii $) 0 \leq y(t) \leq x(t),($ iii $) a(x(t+1)-(1-$ d) $x(t))+e y(t) \leq 1$. A program $\{x(t), y(t)\}$ is simply a program from $x(0)$.

Definition 2 Associated with any program $\{x(t), y(t)\}$ is a gross investment sequence $\{z(t+1)\}$ with $z(t+1) \in \mathbb{R}_{+}^{n}$, and a consumption sequence $\{b y(t)\}$ such that for all $t \in \mathbb{N}, z(t+1)=x(t+1)-(1-d) x(t)$.

Definition 3 A program $\{x(t), y(t)\}$ is called stationary if for all $t \in \mathbb{N},(x(t), y(t))=(x(t+1), y(t+1))$.

We conclude this subsection with a result on the boundedness property of programs.

Proposition 1 For any program $\{x(t), y(t)\}$, there exists $m(x(0)) \in \mathbb{R}_{+}$such that $x(t) \leq m(x(0))$ e for any $t \in \mathbb{N}$.

Proof: The case $t=0$ is a triviality. For $t \in \mathbb{N}_{+}, a x(t) \leq 1+(1-d) a x(t-1) \leq \sum_{\tau=0}^{t-1}(1-d)^{\tau}+(1-$ $d)^{t} a x(0)$. Since $0<d<1$, we obtain $a x(t) \leq(1 / d)+a x(0)$. Let $a_{j}=\min _{1 \leq i \leq n} a_{i}$. Since $a_{i}>0$ for all $i=1,2, \cdots n$, we obtain $x_{i}(t) \leq\left(1 / a_{j}\right)((1 / d)+a x(0)) \equiv m(x(0))$ for all $i=1, \cdots, n$, and complete the proof.

The preferences of the planner are represented by a felicity function, $w: \mathbb{R}_{+} \longrightarrow \mathbb{R}$, which is assumed to be continuous, strictly increasing and concave, and differentiable. ${ }^{29}$ We suppose, as in the literature taking its lead from Ramsey (1928), that future welfare levels are treated like current ones in the planner's objective function. The notion of optimality that we use is due to Brock (1970), and the notion of "overtaking" is due to Atsumi (1965) and von Weiszäcker (1965).30

\footnotetext{
${ }^{27}$ The reader may choose to think of the consumption in period $t$ as the scalar $c(t+1)$, with $c_{i}$ reserved for $b_{i} /\left(1+d a_{i}\right)$, we avoid this notation in the text to prevent any ambiguity.

${ }^{28}$ Note $\{x(t), y(t)\}$ is an abbreviation of $\{x(t), y(t)\}_{t \in \mathbb{N}}$; we use it for notational simplicity.

${ }^{29}$ We leave it to the reader to check that differentiability of $w$ is not needed, and derivatives of $w$ can be replaced uniformly by (for example) the right hand derivative of $w$. These exist since $w$ is concave and the point of evaluation of the (right-hand) derivative is always positive.

${ }^{30}$ Brock (1970) uses the terminology of "weakly maximal" programs for what we call optimal programs. The notion of optimality in Atsumi and von Weiszäcker is stronger, and creates problems in proving existence of optimal programs in many reasonable models.
} 
Definition 4 A program $\left\{x^{*}(t), y^{*}(t)\right\}$ from $x_{o}$ is called optimal if

$$
\liminf _{T \rightarrow \infty} \sum_{t=1}^{T}\left[w(b y(t))-w\left(b y^{*}(t)\right)\right] \leq 0
$$

for every program $\{x(t), y(t)\}$ from $x_{o}$. It is called a stationary optimal program if it is stationary and optimal.

Note that the optimality notion can be restated to say that there does not exist any other program $\{x(t), y(t)\}, x(0)=x_{o}$, a number $\varepsilon>0$ and a time period $t_{\varepsilon}$ such that $\sum_{t=1}^{T}\left[w(b y(t))-w\left(b y^{*}(t)\right)\right]>$ $\varepsilon$ for all $T \geq t_{\varepsilon}$. Thus an optimal program is one in comparison to which no other program from the same initial stock is eventually significantly better, for any given level of significance.

Following McKenzie (1968), we convert the above model into its "reduced form", and as emphasized in the introduction, thereby exploit as far as possible the results of the general theory of intertemporal allocation for our particular case. Define the transition possibility set $\Omega$ as a collection of pairs $\left(x, x^{\prime}\right)$, such that it is possible to obtain the amounts of the $n$ types of machines $x^{\prime}$ in the next period (tomorrow) from the amounts of the $n$ types of machines $x$ available in the current period (today). Formally,

$$
\Omega=\left\{\left(x, x^{\prime}\right) \in \mathbb{R}_{+}^{n} \times \mathbb{R}_{+}^{n}: x^{\prime}-(1-d) x \geq 0 \text { and } a\left(x^{\prime}-(1-d) x\right) \leq 1\right\} .
$$

For any $\left(x, x^{\prime}\right) \in \Omega$, one can consider the amounts $y$ of the $n$ types of machines available for the production of the consumption good. Formally, we have a correspondence $\Lambda: \Omega \longrightarrow \mathbb{R}_{+}^{n}$ given by

$$
\Lambda\left(x, x^{\prime}\right)=\left\{y \in \mathbb{R}_{+}^{n}: 0 \leq y \leq x \text { and } e y \leq 1-a\left(x^{\prime}-(1-d) x\right)\right\} .
$$

For any $\left(x, x^{\prime}\right) \in \Omega$, we shall denote the number of machines that are produced in the period $\left(x^{\prime}-(1-d) x\right)$ by $z$. Note that $z \geq 0$. Finally, the reduced form utility function, $u: \Omega \longrightarrow \mathbb{R}_{+}$, is defined on $\Omega$ such that

$$
u\left(x, x^{\prime}\right)=\max \left\{w(b y): y \in \Lambda\left(x, x^{\prime}\right)\right\} .
$$

We leave it to the reader to check for herself that our assumptions on $w$ imply that the reduced form utility function, $u$, is upper semicontinuous ${ }^{31}$ and concave on $\Omega$, and that it is increasing in its first argument and decreasing in its second argument.

Given the description of the transition possibility set $\Omega$, and of the reduced form utility function, $u$, it is clear that for any program $\{x(t), y(t)\}$ from $x_{o},(x(t), x(t+1)) \in \Omega$ and $y(t) \in \Lambda(x(t), x(t+1))$ for all $t \in \mathbb{N}$. Also, for any optimal program $\left\{x^{*}(t), y^{*}(t)\right\}$ from $x_{o}, w\left(b y^{*}(t)\right)=u\left(x^{*}(t), x^{*}(t+1)\right)$ for all $t \in$ $\mathbb{N}$, and for every program $\{x(t), y(t)\}$ from $x_{o}$,

$$
\liminf _{T \rightarrow \infty} \sum_{t=0}^{T}\left[u(x(t), x(t+1))-u\left(x^{*}(t), x^{*}(t+1)\right)\right] \leq 0 .
$$

\footnotetext{
${ }^{31}$ It is now well understood that continuity of $w$ does not necessarily imply the continuity of $u$; see Dutta-Mitra (1989) for details.
} 
In summary, the basic data of the model denoted by the triple $\left(w,\left(a_{i}, b_{i}\right)_{i=1}^{n}, d\right)$ summarizing the felicity function $w$, the technology $\left(a_{i}, b_{i}\right)_{i=1}^{n}$, and the depreciation rate $d$, is converted to the pair $(u, \Omega)$ summarizing the reduced-form utility function $u$ and the transition possibility set $\Omega$.

\section{The Existence and Uniqueness of a Golden-Rule Stock}

A stationary optimal program is of special significance, and in this section we take the first step in establishing the existence of such a program. We show the existence and uniqueness of a golden-rule stock, and simultaneously, provide a "price support" property of such a stock. ${ }^{32}$ We exploit the concrete structure of the RSS model to provide a purely constructive proof of our claims. This has the additional advantage that we can identify the shadow prices in terms of the basic data of the model.

We begin with a definition.

Definition 5 A golden-rule stock is $\hat{x} \in \mathbb{R}_{+}^{n}$ such that $(\hat{x}, \hat{x})$ is a solution to the problem: maximize $u\left(x, x^{\prime}\right)$ subject to (i) $x^{\prime} \geq x$, (ii) $\left(x, x^{\prime}\right) \in \Omega$.

If we limit ourselves to a stationary program in which only a machine of type $i$ is used and produced, the constraint of labor allows us to maintain the $\operatorname{stock}^{33}\left(1 /\left(1+d a_{i}\right)\right.$ and obtain a stationary consumption stream in the amount $b_{i} /\left(1+d a_{i}\right)=c_{i}$. Since we have assumed (in (1) above) that a machine of type $\sigma$ is the one that uniquely minimizes effective labor costs, we see that it is also the type that uniquely maximizes the consumption per unit of labor. ${ }^{34}$ Denote $\hat{y}=\left(1 /\left(1+d a_{\sigma}\right)\right) e(\sigma)$, and note that if we are in such a stationary state, $b \hat{y}=\left(b_{\sigma} /\left(1+d a_{\sigma}\right)\right)$ and $w^{\prime}(b \hat{y})$ is the marginal utility of output produced. Furthermore, since the labor cost of a machine of type $i$ is $a_{i}$, and a unit of labor is worth $\left(\left(1+d a_{i}\right) / b_{i}\right)^{-1}$ units of output, a machine is worth $a_{i} \times\left(b_{i} /\left(1+d a_{i}\right)\right.$ in terms of output, and $w^{\prime}(b \hat{y})\left(a_{i} \times\left(b_{i} /\left(1+d a_{i}\right)\right)\right)$ in terms of utils. We can then identify a stationary price system $(\hat{q}$ in terms of the consumption good and $\hat{p}$ in terms of utils $)^{35}$ for the various types of machines as $\hat{q}_{i}=\left(a_{i} b_{i} /\left(1+d a_{i}\right)\right)$ and $\hat{p}_{i}=w^{\prime}(b \hat{y}) \hat{q}_{i}$ for each $i=1, \cdots, n$.

We can now present a simple but important result.

Lemma $1 w(b \hat{y}) \geq w(b y)+\hat{p} x^{\prime}-\hat{p} x$ for any $\left(x, x^{\prime}\right) \in \Omega$, and for any $y \in \Lambda\left(x, x^{\prime}\right)$.

Proof: For any $\left(x, x^{\prime}\right) \in \Omega$ and $y \in \Lambda\left(x, x^{\prime}\right)$, we have ${ }^{36}$

$$
\begin{aligned}
b \hat{y}-b y-\hat{q}\left(x^{\prime}-x\right) & =c_{\sigma}-b y-\hat{q}\left(x^{\prime}-x\right) \\
& =c_{\sigma}-b y-\hat{q}\left(x^{\prime}-(1-d) x\right)+d \hat{q} x \\
& =c_{\sigma}(1-e y-a z)+c_{\sigma} e y+c_{\sigma} a z-b y-\hat{q} z+d \hat{q} x
\end{aligned}
$$

\footnotetext{
${ }^{32}$ We show in Section 4 (see Theorem 2) that this golden-rule stock defines a stationary optimal program.

${ }^{33}$ The labor requirements of the consumption sector in the amount $\left(1 /\left(1+d a_{i}\right)\right.$ plus those of the investment sector arising from replacement for depreciation in the amount $d a_{i} /\left(1+d a_{i}\right)$ add up to the total labor available.

${ }^{34}$ As alluded to in Footnote 26 above.

${ }^{35}$ When the felicity function is linear, the magnitudes of $\hat{p}$ and $\hat{q}$ are identical, though their units remain different. Note also the identities $\hat{q}_{i}=a_{i} c_{i}$ and $c_{i}+d \hat{q}_{i}=b_{i}$ for all $i$.

${ }^{36}$ Note that in the derivation of (2) and (3) below, we appeal to the identities referred to in Footnote 35.
} 


$$
\begin{aligned}
& =c_{\sigma}(1-e y-a z)+\sum_{i=1}^{n}\left(c_{\sigma}-b_{i}\right) y_{i}+\sum_{i=1}^{n}\left(c_{\sigma}-c_{i}\right) a_{i} z_{i}+d \hat{q} x \\
& =c_{\sigma}(1-e y-a z)+\sum_{i=1}^{n}\left(c_{\sigma}-c_{i}\right) y_{i}+\sum_{i=1}^{n}\left(c_{\sigma}-c_{i}\right) a_{i} z_{i}+d \hat{q}(x-y)
\end{aligned}
$$

Since $\left(x, x^{\prime}\right) \in \Omega, z \geq 0$. Since $y \in \Lambda\left(x, x^{\prime}\right), x \geq y$ and $1-e y-a z \geq 0$. We can now appeal to our standing hypothesis as described in (1) to assert that

$$
b y-b \hat{y} \leq \hat{q} x-\hat{q} x^{\prime} .
$$

Given our hypotheses on the felicity function $w$, we obtain as a consequence of (4),

$$
w(b y)-w(b \hat{y}) \leq w^{\prime}(b \hat{y})(b y-b \hat{y}) \leq w^{\prime}(b \hat{y})\left(\hat{q} x-\hat{q} x^{\prime}\right)=\left(\hat{p} x-\hat{p} x^{\prime}\right)
$$

A simple transposition of terms completes the proof.

We can now state the principal result of this section. ${ }^{37}$

Theorem 1 There exists a unique golden-rule stock $\hat{x}=\left(1 /\left(1+d a_{\sigma}\right)\right) e(\sigma)$.

Proof: Let $\hat{y}=\hat{x}=\left(1 /\left(1+d a_{\sigma}\right)\right) e(\sigma)$, and check that $(\hat{x}, \hat{x}) \in \Omega$, and $\hat{y} \in \Lambda(\hat{x}, \hat{x})$. Next, appeal to Lemma 1 to assert that $(\hat{x}, \hat{x})$ is a solution to the problem specified in Definition 5 , and hence that $\hat{x}$ is a golden-rule stock.

We can also show that it is a unique solution to this problem. Suppose to the contrary that $\left(\tilde{x}, \tilde{x}^{\prime}\right)$ is another solution with a corresponding $\tilde{y} \in \Lambda\left(\tilde{x}, \tilde{x}^{\prime}\right)$ and $\tilde{z}^{\prime}=\tilde{x}^{\prime}-(1-d) \tilde{x}$. Since $w(\cdot)$ is strictly increasing, $b \tilde{y}=b \hat{y}=c_{\sigma}$. On substituting $\tilde{x}, \tilde{y}$ and $\tilde{z}$ for $x, y$ and $z$ in (3) above, we obtain the fact that the right hand side of (3) equals zero, which implies that each of its four terms is zero. This implies that $\tilde{y}_{i}=0=\tilde{z}_{i}$ for $i \neq \sigma$, that $\tilde{x}_{i}=\tilde{y}_{i}$ for all $i$, and that $\tilde{y}_{\sigma}+a_{\sigma} \tilde{z}_{\sigma}=1$. Coupling the first assertion with the equality $b \tilde{y}=c_{\sigma}$, we obtain that $\tilde{y}_{\sigma}=1 /\left(1+d a_{\sigma}\right)$, and hence from the third assertion that $\tilde{x}=1 /\left(1+d a_{\sigma}\right) e(\sigma)$. From the last assertion we can then obtain that $\tilde{z}_{\sigma}=d /\left(1+d a_{\sigma}\right)$ and hence that $\tilde{x}^{\prime}=\tilde{z}-(1-d) \tilde{x}=\left(d /\left(1+d a_{\sigma}\right)+(1-d) /\left(1+d a_{\sigma}\right)\right) e(\sigma)=\left(1 /\left(1+d a_{\sigma}\right)\right) e(\sigma)$. The demonstration is complete.

\section{The Existence of an Optimal Program}

In this section, we prove the existence of an optimal program from an arbitrarily given initial stock. We follow the methods of Brock (1970) which in turn build on those of Gale (1967) - this methodology relies on the concept of a good program and then exploits the assumption of a unique golden-rule stock to deduce the average turnpike property of such a program. We follow the same conceptual benchmarks in the context of the RSS model and present a unified treatment both to highlight certain steps that are crucial for subsequent argumentation and to avoid possibly confusing cross-referencing. ${ }^{38}$

\footnotetext{
${ }^{37}$ We remind the reader of our standing hypothesis as expressed in (1).

${ }^{38}$ Note that we cannot directly apply the relevant theorems in Gale, Brock (1970) or McKenzie (1968, 1987) since the assumptions of these theorems are not directly satisfied; instead, the concrete structure of the RSS model allows a simplification of the arguments.
} 
Definition 6 A program $\{x(t), y(t)\}$ is called good if there exists $G \in \mathbb{R}$ such that $\sum_{t=0}^{T}(w(b y(t))-$ $w(b \hat{y})) \geq G$ for all $T \in \mathbb{N}$. A program is called bad if $\lim _{T \rightarrow \infty} \sum_{t=0}^{T}(w(b y(t))-w(b \hat{y}))=-\infty$.

Proposition 2 There exists a good program from any arbitrary initial stock $x_{o} \in \mathbb{R}_{+}^{n}$.

Proof: For each $t \in \mathbb{N}$, let $z(t+1)=d \hat{x}$. Define $y(0)=0$, and $y(t+1)=(1-d) y(t)+d \hat{x}$ for $t \in \mathbb{N}$. Then, $y(t)$ is monotonically non-decreasing, and converges to $\hat{x}$ as $t \rightarrow \infty$. Given an arbitrary initial stock, $x_{o}$, define $x(0)=x_{o}$, and for each $t \in \mathbb{N}, x(t+1)=(1-d) x(t)+z(t+1)$. Then, it is easy to check that $\{x(t), y(t)\}$ is a program from $x_{o}$. Given the definition of the sequence $\{y(t)\}$, we have $(b y(t)-b \hat{x})=(1-d)^{t}(b y(1)-b \hat{x})$ for $t \geq 2$, and $b y(t) \geq d b \hat{x}$ for $t \in \mathbb{N}_{+}$. Thus, we have for $t \in \mathbb{N}_{+}$,

$$
[w(b \hat{x})-w(b y(t))] \leq w^{\prime}(b y(t))(b \hat{x}-b y(t)) \leq w^{\prime}(d b \hat{x})(b \hat{x}-b y(1))(1-d)^{t-1} .
$$

Thus, the sequence $\{w(b \hat{x})-w(b y(t))\}$ is summable, and so $\{x(t), y(t))\}$ is a good program from $x_{o}$.

Proposition 3 For any program $\{x(t), y(t)\}$, there exists $M(x(0)) \in \mathbb{R}_{+}$such that for any $t_{1} \in \mathbb{N}$, and any integer $t_{2} \geq t_{1}, \sum_{t=t_{1}}^{t_{2}}(w(b y(t))-w(b \hat{y})) \leq M(x(0))$.

Proof: From Lemma 1, for any $t_{2} \geq t_{1}, \sum_{t=t_{1}}^{t_{2}} w(b y(t))-w(b \hat{y}) \leq \hat{p}\left(x\left(t_{1}\right)-x\left(t_{2}+1\right)\right) \leq \hat{p} x\left(t_{1}\right) \leq$ $m(x(0)) \sum_{j=1}^{n} \hat{p}_{j}$. Let $M(x(0))=m(x(0)) w^{\prime}\left(b_{\sigma} / 1+d a_{\sigma}\right) \sum_{j=1}^{n} a_{i} b_{i} /\left(1+d a_{i}\right)$ to complete the proof.

Proposition 4 Any program that is not good is bad.

Proof: For any program $\{x(t), y(t)\}$ that is not good, and for any $N \in \mathbb{R}$, there exists $T_{N}$ such that $\sum_{\tau=0}^{T_{N}}(w(b y(\tau))-w(b \hat{y})) \leq N-M(x(0)), M(x(0))$ the real number whose existence is asserted in Proposition 3. By choosing $t_{1}=T_{N}+1$ and $t_{2}=t>T_{N}+1$ in Proposition 3, we obtain that $\sum_{\tau=T_{N}+1}^{t}(w(b y(\tau))-w(b \hat{y})) \leq M(x(0))$ for all $t>T_{N}+1$. On adding these two expressions, we obtain that $\sum_{\tau=0}^{t}(w(b y(\tau))-w(b \hat{y})) \leq N$ for all $t>T_{N}$, and complete the proof.

Definition 7 A program $\{x(t), y(t)\}$ exhibits the average turnpike property if $\lim _{T \rightarrow \infty}(\bar{x}(T), \bar{y}(T))$ $=(\hat{x}, \hat{y})$, where $\bar{x}(T)=(1 / T) \sum_{t=0}^{T-1} x(t)$ and $\bar{y}(T)=(1 / T) \sum_{t=0}^{T-1} y(t)$ for all $T \in \mathbb{N}_{+}$.

The proofs of Propositions 5, 8 and 9 are technical, and we relegate them to the Appendix.

Proposition 5 Every good program exhibits the average turnpike property.

For any $y \in \Lambda\left(x, x^{\prime}\right)$ and any $\left(x, x^{\prime}\right) \in \Omega$, let

$$
\delta_{(\hat{x}, \hat{p})}\left(x, x^{\prime}\right)=w(b \hat{y})-w(b y)-\hat{p}\left(x^{\prime}-x\right)=\hat{p}\left(x-x^{\prime}\right)-(w(b y)-w(b \hat{y})) .
$$

Whenever there is no possibility of confusion, we shall abbreviate $\delta_{(\hat{x}, \hat{p})}(x(t), x(t+1))$ by $\delta(t)$ for any program $\{x(t), y(t)\}$. We shall refer to $\{\delta(t)\}$ as the value-loss sequence associated with the program $\{x(t), y(t)\}$. 
Proposition 6 The value-loss sequence $\{\delta(t)\}_{t \in \mathbb{N}}$ of any program $\{x(t), y(t)\}$ is non-negative, and

$$
\sum_{t=0}^{T}(w(b y(t))-w(b \hat{y}))=\hat{p}(x(0)-x(T+1))-\sum_{t=0}^{T} \delta(t) \text { for all } T \in \mathbb{N} \text {. }
$$

Proof: For each $t \in \mathbb{N}$, let $\delta(t)=\hat{p}(x(t)-x(t+1))-(w(b y(t))-w(b \hat{y}))$. Since $\{x(t), y(t)\}$ is a program, we can appeal to Lemma 1 to assert that $\delta(t) \geq 0$ for all $t \in \mathbb{N}$. On summing over $t$, and rearranging, we complete the proof of the assertion.

We now define the aggregate value-loss associated with any program as

$$
\Delta\left(x_{o}\right)=\inf \left\{\sum_{t=0}^{\infty} \delta(t):\{x(t), y(t)\} \text { is a program from } x_{o}\right\} .
$$

Our next two results assert that this infimum is a finite number and that it can be attained.

Proposition 7 The value-loss sequence $\{\delta(t)\}_{t \in \mathbb{N}}$ of any program $\{x(t), y(t)\}$ is summable if and only if it is good. Hence $\lim _{t \rightarrow \infty} \delta(t)=0$ for any good program.

Proof: For any good program, Proposition 6 allows us to assert the existence of $G \in \mathbb{R}$ such that for all $t \in \mathbb{N}$,

$$
\begin{aligned}
\sum_{t=0}^{T} \delta(t) & =\hat{p}(x(0)-x(T+1))-\sum_{t=0}^{T}(w(b y(t))-w(b \hat{y})) \\
& \leq \hat{p}(x(0)-x(T+1))-G \leq \hat{p}(x(0))-G .
\end{aligned}
$$

Since $\sum_{t=0}^{\infty} \delta(t)$ is a finite number, certainly $\lim _{t \rightarrow \infty} \delta(t)=0$. On the other hand, the first equality and Proposition 1 allows us to assert that a program with a summable value-loss sequence is good.

Proposition 8 There exists a program $\left\{x^{\prime}(t), y^{\prime}(t)\right\}$ from an arbitrary initial stock $x_{o}$ such that its associated value-loss sequence $\left\{\delta^{\prime}(t)\right\}$ satisfies $\sum_{t=0}^{\infty} \delta^{\prime}(t)=\Delta\left(x_{o}\right)$ where $0 \leq \Delta\left(x_{o}\right)<\infty$.

Proposition 9 A program $\{x(t), y(t)\}$ whose associated value-loss sequence $\{\delta(t)\}$ satisfies $\sum_{t=0}^{\infty} \delta(t)=$ $\Delta(x(0))$ is optimal.

Theorem 2 For any arbitrary initial stock, $x_{o} \in \mathbb{R}_{+}^{n}$, there exists an optimal program from $x_{o}$. If the initial stock $x_{o}$ equals $\hat{x}=\hat{y}=\left(1 /\left(1+d a_{\sigma}\right)\right) e(\sigma)$, then the stationary program $\{\hat{x}, \hat{y}\}$ is an optimal program from $x_{o}$.

Proof: Proposition 9 guarantees that the program whose existence is asserted in Proposition 8 is optimal. For the second claim, simply note that the aggregate value-loss of the stationary program is (trivially) zero and that an appeal to Proposition 9 completes the argument. 


\section{Choice of Techniques in the Long-Run}

We are now in a position to describe what the economy looks like in the long-run. Towards this end, we begin with a characterization of the von Neumann facet as described in McKenzie (1968, 1986). It is of interest that under our standing hypothesis as described in (1), this reduces to a line in the Euclidean space of dimension $2 n$.

Lemma 2 The von Neumann facet $\left\{\left(x, x^{\prime}\right) \in \Omega: \delta_{(\hat{p}, \hat{x})}\left(x, x^{\prime}\right)=0\right\}$ is a subset of $\left\{\left(x, x^{\prime}\right) \in \Omega: x_{i}^{\prime}=\right.$ $\left.x_{i}=0, i \neq \sigma, x_{\sigma}^{\prime}=\left(1 / a_{\sigma}\right)+\xi_{\sigma} x_{\sigma}\right\}, \xi_{\sigma}=1-d-\left(1 / a_{\sigma}\right)$, with equality if the felicity function $w$ is linear. If the felicity function is strictly concave, the facet is the singleton $\{(\hat{x}, \hat{x})\}$.

Proof: Pick $\left(\tilde{x}, \tilde{x}^{\prime}\right) \in \Omega$ and $\tilde{y} \in \Lambda\left(\tilde{x}, \tilde{x}^{\prime}\right)$ such that $\delta_{(\hat{x}, \hat{p})}\left(\tilde{x}, \tilde{x}^{\prime}\right)=0$. From (5) we obtain $w(b \tilde{y})-w(b \hat{y})+$ $\hat{p}\left(\tilde{x}^{\prime}-\tilde{x}\right)=0$. On appealing to the concavity of $w(\cdot)$, this reduces to

$$
w(b \tilde{y})-w(b \hat{y}) \leq w^{\prime}(b \hat{y})(b \tilde{y}-b \hat{y}) \Longrightarrow b \hat{y}-b \tilde{y}-q\left(\tilde{x}^{\prime}-\tilde{x}\right) \leq 0 .
$$

This combined with (4) and (3) yields

$$
c_{\sigma}(1-e \tilde{y}-a \tilde{z})+\sum_{i=1}^{n}\left(c_{\sigma}-c_{i}\right) \tilde{y}_{i}+\sum_{i=1}^{n}\left(c_{\sigma}-c_{i}\right) a_{i} \tilde{z}_{i}+d q(\tilde{x}-\tilde{y})=0 .
$$

This implies that $\tilde{z}_{i}=0=\tilde{y}_{i}=\tilde{x}_{i}=\tilde{x}_{i}^{\prime}$ for all $i \neq \sigma$. Furthermore, that $\tilde{y}_{\sigma}=\tilde{x}_{\sigma}$ and that

$$
\tilde{y}_{\sigma}-a_{\sigma} \tilde{z}_{\sigma}=1 \Longrightarrow \tilde{x}_{\sigma}+a_{\sigma}\left(\tilde{x}_{\sigma}^{\prime}-(1-d) \tilde{x}_{\sigma}\right)=1 \Longrightarrow \tilde{x}_{\sigma}^{\prime}=\left(1 / a_{\sigma}\right)+\xi_{\sigma} \tilde{x}_{\sigma}
$$

Now suppose that $w(\cdot)$ is strictly concave and that $b \tilde{y} \neq b \hat{y}$. We then obtain a strict inequality in (6) and thereby contradict (4). Thus $b \tilde{y}=b \hat{y}=c_{\sigma}$. On appealing to the computations above, we obtain that $\tilde{y}_{\sigma}=1 /\left(1+d a_{\sigma}\right)=\tilde{x}_{\sigma}$, and hence that $\tilde{x}_{\sigma}^{\prime}=\left(1 / a_{\sigma}\right)+\xi_{\sigma} \tilde{x}_{\sigma}=1 /\left(1+d a_{\sigma}\right)$.

For the reverse implication in the linear case, pick $\left(x, x^{\prime}\right) \in \Omega$ such that $x_{\sigma}^{\prime}=\left(1 / a_{\sigma}\right)+\xi_{\sigma} x_{\sigma}$, $x_{i}^{\prime}=x_{i}=0, i \neq \sigma$, and $y_{\sigma}=x_{\sigma}$. On substituting these values in the left hand side of (3), we see that it is equal to zero. But that is precisely $\delta_{(\hat{x}, \hat{p})}(x, x)$ in the linear case.

Before we present the principal result of this section, we record the following observation.

\section{Proposition 10 Any optimal program is good.}

Proof: Let $\{x(t), y(t)\}$ be an optimal program, and suppose it is not good. By Proposition 2, there exists a good program $\left\{x^{\prime}(t), y^{\prime}(t)\right\}$ starting from $x(0)$. Hence there exists $G \in \mathbb{R}$ such that for all $T \in \mathbb{N}_{+}, \sum_{t=0}^{T}\left(w\left(b y^{\prime}(t)\right)-w(b \hat{y})\right) \geq G$. Pick any $\varepsilon>0$, and appeal to Proposition 4 to guarantee the existence of $t_{\varepsilon}$ such that $\sum_{t=0}^{T}(w(b y(t))-w(b \hat{y}))<G-\varepsilon$ for all $T \geq t_{\varepsilon}$. Putting these two expressions together, we obtain that $\sum_{t=0}^{T}\left(w\left(b y^{\prime}(t)\right)-w(b y(t))\right)>\varepsilon$ for all $T \geq t_{\varepsilon}$, and hence a contradiction to the fact that $\{(x(t), y(t)\}$ is an optimal program.

We can now present 
Theorem 3 Any optimal program $\{x(t), y(t)\}$ converges to the von Neumann facet, and thus $\lim _{t \rightarrow \infty} x_{i}(t)=\lim _{t \rightarrow \infty} y_{i}(t)=\lim _{t \rightarrow \infty} z_{i}(t)=0$ for all $i \neq \sigma$. If the felicity function $w(\cdot)$ is strictly concave, $\lim _{t \rightarrow \infty} x(t)=\lim _{t \rightarrow \infty} y(t)=\left(1 / 1+d a_{\sigma}\right) e(\sigma)$ and $\lim _{t \rightarrow \infty} z(t)=\left(d a_{\sigma} / 1+d a_{\sigma}\right) e(\sigma)$.

Proof: Suppose that there exists $\varepsilon>0$ such that for all $k \in \mathbb{N}_{+}$, there exists $t(k) \geq k$ such that $\sum_{i \neq \sigma}\|x(t(k))\|>\varepsilon$. We can then assert that for the value-loss sequence $\{\delta(t(k))\}_{k \in \mathbb{N}_{+}}$, there exists $\delta_{o}>0$ and $k_{o} \in \mathbb{N}_{+}$such that for all $k \geq k_{o}, \delta(t(k)) \geq \delta_{o}$. If the assertion is valid, we obtain a contradiction to Proposition 7 and complete the proof of the first claim. Thus, suppose that the assertion is false. Then we can manufacture a sequence of integers $\left\{k_{i}\right\}_{i \in \mathbb{N}_{+}}$such that $\lim _{i \rightarrow \infty} \delta\left(t\left(k_{i}\right)\right)=0$. Now consider the sequence $\left\{\left(x\left(t\left(k_{i}\right)\right), x\left(t\left(k_{i}\right)+1\right)\right)\right\}_{i \in \mathbb{N}_{+}}$and appeal to Proposition 1 to guarantee the existence of a subsequence that converges to a point $\left(\tilde{x}, \tilde{x}^{\prime}\right)$. Since $\Omega$ is closed, and $w(\cdot)$ is continuous, $\delta_{(\hat{p}, \hat{x})}\left(\tilde{x}, \tilde{x}^{\prime}\right)=0$. We now appeal to Lemma 2 to obtain a contradiction to our initial hypothesis.

For the case of a strictly concave felicity function, repeat the argument above but with $\| x(t(k))-$ $\hat{x}\|+\| x(t(k+1))-\hat{x} \|>\varepsilon$. In this case, $\left(\tilde{x}, \tilde{x}^{\prime}\right)=(\hat{x}, \hat{x})$, and we again appeal to Lemma 2 to obtain a contradiction to our initial hypothesis.

\section{Choice of Techniques in Transition}

In this section, we turn to the question of which machines are optimally used and produced - the choice of techniques - not only in the long-run, but also in the medium- to short-run. Our discussion revolves around the identification and formalization of a policy prescribed in Stiglitz (1968). Thus, for the case of a linear felicity function, we present simple examples of economies, consisting of only a single type of machine and thereby posing no issue as to the choice of technique in production, ${ }^{39}$ in which consumption and capital stock exhibit a two-period cycle along an optimal program or a four-period cycle along a Stiglitz program, which is thereby shown to be bad. The first shows the optimality of periodic overbuilding and over-consuming relative to the golden-rule levels, and the second, the non-optimality of a no excess-capacity policy - phenomena that Stiglitz (1968) apparently does not encounter in his continuoustime formulation of the model. ${ }^{40}$ Leaving aside questions relating to the utilization of machines, and focussing only on their production, we also present an example of an economy consisting of two types of machines in which a machine other than the golden-rule machine is produced in the very first period along an optimal program. For a non-linear felicity function, this establishes the non-optimality of a Stiglitz production program (Definition 9 below), and gives an affirmative answer to the question as to whether there is a compelling reason to ever produce a type of machine which we know would eventually be depreciated to zero? ${ }^{41}$

\footnotetext{
${ }^{39}$ The question of the choice of technique in terms of use of course remains: should all of the stocks of the machine be utilized until production is undertaken? This question is investigated in Section 6.3 below.

${ }^{40}$ It is worth re-emphasizing in this connection that Brock's 1970 results were not available to Stiglitz in 1968.

${ }^{41}$ Stiglitz (1973, pp. 146-148) has an example of a four machine economy where such a phenomenon can occur, but it is with discounting and a minimum consumption constraint. In this work, Stiglitz is primarily interested in what he calls the phenomena of "recurrence" - a situation when a machine once in service is put out of service to be brought back into service again.
} 


\subsection{Stiglitz' Policy Prescriptions}

We present Stiglitz' policy prescriptions in the form of particular programs. Towards this end, let $D=\left\{i \in\{1, \cdots, n): b_{i} \geq c_{\sigma}=b_{\sigma} /\left(1+d a_{\sigma}\right)\right\}$ be the set of machine-types whose output per unit labor ratios are not less than the effective output per unit labor ratio of machines of type $\sigma .{ }^{42}$ We shall refer to such types as desirable and to those not in $D$ as undesirable. Under a Stiglitz policy, labor is allocated in each time period to a set of available desirable machines with a higher type of machine having a priority over a lower one, ${ }^{43}$ and any remaining labor allocated towards producing only one type of machine, that delineated by $\sigma$. More formally,

Definition 8 A program $\{x(t), y(t)\}$ with an associated gross investment sequence $\{z(t+1)\}$ is said to be a Stiglitz program if for any $t \in \mathbb{N}$ the following policy prescriptions are followed.

(i) If $0 \leq \sum_{i \in D} x_{i}(t) \leq 1$, let $y_{i}(t)=x_{i}(t)$ for all $i \in D$, $y_{i}(t)=0$ for all $i \notin D$, and $z(t+1)=$ $\left(\left(1-\sum_{i \in D} x_{i}(t)\right) / a_{\sigma}\right) e(\sigma)$.

(ii) If $\sum_{i \in D} x_{i}(t)>1$ and $x_{1}(t) \geq 1$, let $y(t)=e(1)$ and $z(t+1)=0$.

(iii) If $\sum_{i \in D} x_{i}(t)>1$ and $x_{1}(t)<1$, let $y_{i}(t)=x_{i}(t)$ for all $i=1, \cdots, i_{o}-1, y_{i_{o}}(t)=1-\sum_{i=1}^{i_{o}-1} x_{i}(t)$, where $i_{o} \in D$ such that $\sum_{i=1}^{i_{o}-1} x_{i}(t)<1$ and $\sum_{i=1}^{i_{o}} x_{i}(t) \geq 1$, and $z(t+1)=0$.

It is perhaps uncontroversial that the crucial aspect of the issue of choice of technique relates to production rather than the utilization of the "correct" type of machine. In keeping with this, we are also interested in the following kind of programs which contain, as a strict subset, the set of Stiglitz programs.

Definition 9 A program $\{x(t), y(t)\}$ with an associated gross investment sequence $\{z(t+1)\}$ is said to be a Stiglitz production program if for any $t \in \mathbb{N}, z_{i}(t+1)=0$ for all $i \neq \sigma$.

In a continuous-time framework of our model, with a linear felicity function, Stiglitz (1968) has argued that an optimal program must follow the policy prescriptions described above. This result turns out to be invalid in our framework, and we provide three examples in the next three subsections to illustrate this observation. In Section 7, we provide alternate sets of sufficient conditions under which the Stiglitz' assertion is valid in our framework.

\subsection{Non-Monotonicity of an Optimal Program}

Stiglitz (1968, p.607) notes that an implication of his policy prescriptions is that employment and output in the consumption good sector increases monotonically, if the economy is initially capital poor. Our first example shows that such a monotonicity property is invalid in general in our framework.

\footnotetext{
${ }^{42}$ See Footnote 26 and the associated text. Note that $\sigma \in D$.

${ }^{43}$ Recall that without any loss of generality, the machine types have been numbered so that $b_{i} \geq b_{i+1}$ for all $i=1, \cdots, n$.
} 
We present an example of an economy with a linear felicity function in which the optimal path cycles around the golden rule stock. The economy has available to it only one type of machine whose (labor) input and output coefficients $\left(a_{1}, b_{1}\right)$ are given by $(2 / 3,1)$, the depreciation rate $d$ by $1 / 2$, and the felicity function by $w(b y)=y$. The reduced form of the economy is given by:

$$
\begin{aligned}
\Omega & =\left\{\left(x, x^{\prime}\right) \in \mathbb{R}_{+}^{2}:(1 / 2) x+(3 / 2) \geq x^{\prime} \geq(1 / 2) x\right\}, \\
\Lambda\left(x, x^{\prime}\right) & =\left\{y \in \mathbb{R}_{+}: y \leq x \text { and } y \leq 1+(1 / 3)\left(x-2 x^{\prime}\right)\right\}=\left\{y \in \mathbb{R}_{+}: y \leq \min \left[1+(1 / 3)\left(x-2 x^{\prime}\right), x\right]\right\}, \\
u\left(x, x^{\prime}\right) & =\max \left\{w(b y): y \in \Lambda\left(x, x^{\prime}\right),\left(x, x^{\prime}\right) \in \Omega\right\}=\min \left[1+(1 / 3)\left(x-2 x^{\prime}\right), x\right] .
\end{aligned}
$$

Consider the program $\{x(t), y(t)\}$ given by $x(t)=y(t)=3 / 4$, with a gross investment of $z(t+1)=$ $x(t+1)-(1-d) x(t)=3 / 4-(1 / 2)(3 / 4)=3 / 8$, for all $t \in N$. We claim that this is a stationary optimal program from $x(0)=3 / 4$. Towards this end, we show that $(3 / 4,3 / 4)$ is the unique solution to the problem delineated in Definition 5, and hence that $3 / 4$ is the unique golden-rule stock.

First observe that $u(3 / 4,3 / 4)=\min [1-(1 / 3)(3 / 4), 3 / 4]=3 / 4$, and that $u\left(x, x^{\prime}\right)=\min [1-$ $\left.(1 / 3) x-(2 / 3)\left(x^{\prime}-x\right), x\right]$. Now if $0 \leq x<3 / 4, u\left(x, x^{\prime}\right)<(3 / 4)$. And if $x>3 / 4$, then $x^{\prime} \geq x$ implies $1-(1 / 3) x-(2 / 3)\left(x^{\prime}-x\right) \leq 1-(1 / 3) x<3 / 4$. Hence, $u\left(x, x^{\prime}\right)<(3 / 4)=u(3 / 4,3 / 4)$, and the argument is complete.

Next, consider a program such that $y(t)=x(t)$ for all $t \in \mathbb{N}, x(t)=1 / 2$ for all even $t \in \mathbb{N}$, and $x(t)=1$ for all odd $t \in \mathbb{N}$. It easy to check that this is a program that starts from $1 / 2$ and oscillates around $3 / 4$. All that we need to show is that it is an optimal program starting from $1 / 2$. Towards this end, we note that $\hat{p}=\hat{q}=1 / 2$, and that this program makes a zero value-loss in each period at these prices:

$$
\delta(t)= \begin{cases}(1 / 2)+(1 / 2)-(1 / 2)(1 / 2)-3 / 4=0 & \text { for } t=0,2, \cdots \\ 1+(1 / 2)(1 / 2)-(1 / 2)-3 / 4=0 & \text { for } t=1,3, \cdots\end{cases}
$$

An appeal to Proposition 9 then completes the argument.

\subsection{Non-Optimality of a Stiglitz Program}

It can be easily checked in the example of the previous subsection that the cyclic optimal program is a Stiglitz program. In this subsection, we ask whether the set of optimal programs is identical to the set of Stiglitz programs, and perhaps surprisingly discover this to be decisively not the case. We present an example of a simple economy with a linear felicity function in which at a particular initial stock, the unique Stiglitz program is bad, leave alone optimal.

The economy has available to it only one type of machine whose (labor) input and output coefficients $\left(a_{1}, b_{1}\right)$ are given by $(2 / 5,1)$, the depreciation rate $d$ by $1 / 2$, and the felicity function by $w(b y)=y$. The reduced form of the economy is given by:

$$
\Omega=\left\{\left(x, x^{\prime}\right) \in \mathbb{R}_{+}^{2}:(1 / 2) x+(5 / 2) \geq x^{\prime} \geq(1 / 2) x\right\},
$$


$\Lambda\left(x, x^{\prime}\right)=\left\{y \in \mathbb{R}_{+}: y \leq x\right.$ and $\left.y \leq 1+(1 / 5)\left(x-2 x^{\prime}\right)\right\}=\left\{y \in \mathbb{R}_{+}: y \leq \min \left[1+(1 / 5)\left(x-2 x^{\prime}\right), x\right]\right\}$, $u\left(x, x^{\prime}\right)=\max \left\{w(b y): y \in \Lambda\left(x, x^{\prime}\right),\left(x, x^{\prime}\right) \in \Omega\right\}=\min \left[1+(1 / 5)\left(x-2 x^{\prime}\right), x\right]$.

Consider the program $\{x(t), y(t)\}$ given by $x(t)=y(t)=5 / 6$, with a gross investment of $z(t+1)=$ $x(t+1)-(1-d) x(t)=5 / 6-(1 / 2)(5 / 6)=5 / 12$, for all $t \in N$. We claim that this is a stationary optimal program from $x(0)=5 / 6$. Towards this end, we show that $(5 / 6,5 / 6)$ is the unique solution to the problem delineated in Definition 5 , and hence that $5 / 6$ is the unique golden-rule stock.

First observe that $u(5 / 6,5 / 6)=\min [1-(1 / 5)(5 / 6), 5 / 6]=5 / 6$, and that $u\left(x, x^{\prime}\right)=\min [1-$ $\left.(1 / 5) x-(2 / 5)\left(x^{\prime}-x\right), x\right]$. Now if $0 \leq x<5 / 6, u\left(x, x^{\prime}\right)<(5 / 6)$. And if $x>5 / 6$, then $x^{\prime} \geq x$ implies $1-(1 / 5) x-(2 / 5)\left(x^{\prime}-x\right) \leq 1-(1 / 5) x<5 / 6$. Hence, $u\left(x, x^{\prime}\right)<(5 / 6)=u(5 / 6,5 / 6)$, and the argument is complete.

Next, consider a program such that for all $t \in \mathbb{N}, x(4 t)=1=y(4 t), x(4 t+1)=1 / 2=$ $y(4 t+1), x(4 t+2)=3 / 2, y(4 t+2)=1, x(4 t+3)=3 / 4=y(4 t+3)$. It easy to check that this is a program that starts from 1 and returns to it after four periods. It is also easy to see that it is a unique Stiglitz program starting from 1 . In terms of Definition $8, D=\{1\}$, and in three of the four periods of the 4-period cycle, condition (ii) applies and usage and production levels are uniquely set to maintain full employment and no excess capacity. In other words, in these periods, all of the desirable machines are utilized, and all of the remaining labor (none in one of the 3 periods) is allocated to the production of new machines. In the one remaining period, $4 t+2$, there is full employment but also excess capacity.

It is easy to check that $u(1,1 / 2)=1, u(1 / 2,3 / 2)=1 / 2, u(3 / 2,3 / 4)=1$, and $u(3 / 4,1)=3 / 4$.

Hence for all $n \in \mathbb{N}_{+}, \sum_{t=0}^{4 n}[u(x(t), x(t+1))-(5 / 6)]=-(1 / 12) n$, so that the Stiglitz program is bad. From Proposition 10, we can then conclude that the Stiglitz program is not optimal.

Since there is a unique Stiglitz program starting from $x(0)=1$, the optimal program from $x(0)=1$, which exists by virtue of Theorem 2 , is not a Stiglitz program.

\subsection{Non-Optimality of a Stiglitz Production Program}

We know from the example presented in the previous subsection that an optimal program is not in general a Stiglitz program. In this section, we ask whether every optimal program is at least a Stiglitz production program. Note that in an economy with only one type of machine (as in the examples of the previous two subsections) an optimal program is trivially a Stiglitz production program. Thus, we need to consider economies with at least two types of machines for the question to be non-trivial. We present an example of an economy with two types of machines and a piece-wise linear felicity function in which the machine different from the golden-rule machine is produced in transition along an optimal program.

Consider an economy in which there are two types of machines $(n=2)$ with input coefficients vector given by $a=(2,3)$, output coefficients vector by $b=(4,5)$ and the depreciation rate, $d$, by 0.45 
$(m \equiv(1-d)=0.55)$. Note that

$$
\frac{b_{1}}{a_{1}}=2>(5 / 3)=\frac{b_{2}}{a_{2}} \text { and } \frac{b_{1}}{\left(1+d a_{1}\right)} \approx 2.1052<2.1276 \approx \frac{b_{2}}{\left(1+d a_{2}\right)},
$$

and thus $\sigma=2$ : machines of type 2 constitute the golden-rule stock. The social welfare function, $w$, is defined as follows:

$$
w(y)= \begin{cases}y-2 & \text { for } y \geq 2 \\ 1000(y-2) & \text { for } 0 \leq y<2\end{cases}
$$

The initial stock of machines is specified as $x_{o}=(0.5,0)$.

We know from the analysis of Section 5 that in the long-run only machines of type 2 will be produced and used along an optimal program. We are interested in demonstrating that machines of type 1 will nevertheless be produced in some time period along an optimal program from $x_{o}$. Our method of demonstrating this is to suppose, on the contrary, that machines of type 1 are not produced in period 1 along an optimal program. We show that a consequence of this is that an optimal program will suffer a large disutility (negative utility, large in absolute value) in either the first or the second period of consumption, which results in a large disutility even in the long-run. We construct a program from $x_{o}$ which produces machines of type 1 initially, and reaches the golden-rule stock in a finite number (specifically, eight) of periods; it has non-negative utility in all periods, and, of course, (positive) goldenrule utility from period nine onwards. This shows that our hypothesis that machines of type 1 are not produced on the optimal program in period 1 must be false, and completes the demonstration. We relegate the computational details to the Appendix.

In conclusion to this section, note that the felicity $w$ used in the above example is non-linear, but not strictly concave. We can check that all the calculations shown in the Appendix below remain valid with a strictly concave $w$ defined as follows:

$$
w(y)= \begin{cases}(53 / 47) 2(y-2) /(y-1) & \text { for } y \geq 2 \\ 1000(y-2)-0.5(y-2)^{2} & \text { for } 0 \leq y<2\end{cases}
$$

\section{Sufficient Conditions for the Optimality of the Stiglitz Policy}

The three examples presented in Section 6 show that an optimal program in our framework does not always follow the policy prescriptions of Stiglitz (1968). However, we can provide sufficient conditions under which it does. This section is devoted to presenting results along this line. In the first subsection we consider a linear felicity function, and in a subsequent section, turn to the general case.

\subsection{The Case of a Linear Felicity Function}

The point to be noted about the two examples presented above (in subsections 6.2 and 6.3 ) is the particular value of the parameter $1-d-\left(1 / a_{1}\right)$. We have already referred to this parameter in Section 5 as $\xi_{1}$, and it takes the value -1 in the example in Section 6.2, and the value -2 in the example in Section 
6.3. The sufficient condition for the optimal choice of technique that we present in this section then ${ }^{44}$ requires that $\xi_{\sigma} \geq-1$.

Theorem 4 With a linear felicity function $w$, and with $1>\xi_{\sigma} \geq-1$, any Stiglitz program is an optimal program. ${ }^{45}$

This theorem is a consequence of the following lemma.

Lemma 3 With a linear felicity function $w$, and with $1>\xi_{\sigma} \geq-1$, the aggregate value-losses of any Stiglitz program starting from $x(0)$ equal $\Delta(x(0))$.

The proof of Lemma 3 relies crucially on the sources of value-loss already identified in the proof of Lemma 1. On rewriting (2), we obtain

$$
\begin{aligned}
\delta(t) & =b \hat{y}-b y(t)-\hat{q}(x(t+1)-x(t)) \\
& =c_{\sigma}(1-e y(t)-a z(t+1))+\sum_{i=1}^{n}\left(c_{\sigma}-b_{i}\right) y_{i}(t)+\sum_{i=1}^{n}\left(c_{\sigma}-c_{i}\right) a_{i} z_{i}(t+1)+d q x(t) \\
& =\alpha(t)+\sum_{i \in D}\left(c_{\sigma}-b_{i}\right) y_{i}(t)+\sum_{i \notin D}\left(c_{\sigma}-b_{i}\right) y_{i}(t)+\sum_{i=1}^{n}\left(c_{\sigma}-c_{i}\right) a_{i} z_{i}(t+1)+d q x(t)
\end{aligned}
$$

where $\alpha(t)=c_{\sigma}(1-e y(t)-a z(t+1))$ is the value-loss from unemployment. ${ }^{46}$ This is a five-fold decomposition ${ }^{47}$ of the value-loss at any time-period: the other four terms concern value losses from incorrect usage and incorrect investment. The proof can now be executed by the comparison, period by period, of the magnitudes of the value-losses of the Stiglitz program and those of any other candidate program. We relegate the details to the Appendix, and turn to a

Proof of Theorem 4: Let $\left\{x^{s}(t), y^{s}(t)\right\}$, with an associated value-loss sequence $\left\{\delta^{s}(t)\right\}$, be a Stiglitz program. Since only one type of machine $\sigma$ is constructed under a Stiglitz' policy, and since $\xi_{\sigma} \geq-1$, we can appeal to Lemma 3 to assert that the Stiglitz program is a good program, and (by Proposition $5)$ exhibits the average turnpike property. In order to complete the proof, we need to establish that the hypotheses of Proposition 9 are satisfied, which is to say that $\left\{\delta^{s}(t)\right\}$ satisfies $\sum_{t=0}^{\infty} \delta^{s}(t)=\Delta(x(0))$. An appeal to Lemma 3 then completes the proof.

Next we ask whether, under the conditions identified in Theorem 4, a Stiglitz program is uniquely optimal. Towards this end, we can present

Theorem 5 With a linear felicity function $w$, and with $-1<\xi_{\sigma}<1$, any optimal program $\{x(t), y(t)\}$ is a Stiglitz program.

\footnotetext{
${ }^{44}$ Note that by default $D=\{1\}=\{\sigma\}$ in each of the one-machine examples considered above.

${ }^{45}$ Note that $\xi_{\sigma}<1$.

${ }^{46}$ Introduced only for the typographical reason of reducing the length of the expression below.

${ }^{47}$ For a general discussion of the importance of decomposition principles across time in problems of intertemporal resource allocation, see Bliss (1975, Chapter 6).
} 
Before considering the proof of this theorem, we draw the reader's attention to the fact that unlike Theorem 4 , Theorem 5 does not cover the case $\xi_{\sigma}=-1$. Indeed, Theorem 5 is false for this case. For the economy discussed in Section 6.2, there exists an optimal program (not discussed in subsection 6.2 ) which is not a Stiglitz program. ${ }^{48}$ Thus, we need to rule out the case when the optimal program stays in the von Neumann facet but does not converge to the golden-rule values. Towards this end we can present a result which strengthens the conclusions of Theorem 3 in the case of a linear felicity function and also shows them to hold for a Stiglitz program.

Proposition 11 With a linear felicity function $w$, and with $-1<\xi_{\sigma}<1$, for a program $\{x(t), y(t)\}$ that is either an optimal or a Stiglitz program, $\lim _{t \rightarrow \infty} y_{i}(t)=\lim _{t \rightarrow \infty} z_{i}(t)=0$ for all $i \neq \sigma$, and $\lim _{t \rightarrow \infty} y_{\sigma}(t)=\lim _{t \rightarrow \infty} x_{\sigma}(t)=\hat{x}_{\sigma}=1 /\left(1+d a_{\sigma}\right), \lim _{t \rightarrow \infty} z_{\sigma}(t)=d /\left(1+d a_{\sigma}\right)$.

We relegate the computational details to the Appendix, and turn to a sharpening of Lemma 3.

Lemma 4 In a setting with a linear felicity function $w$, and $-1 \leq \xi_{\sigma}<1$, let $\{\delta(t)\}$ be the valueloss sequence of a program that is not a Stiglitz program and $\left\{\delta^{s}(t)\right\}$ the value-loss sequence of a Stiglitz program starting from the same initial stock. Then there exists $\varepsilon>0$ such that $\sum_{t=0}^{\infty} \delta(t)-\sum_{t=0}^{\infty} \delta^{s}(t)>$ $\varepsilon$.

We indicate in the Appendix how the proof of Lemma 4 is a straightforward modification of the computations presented in the proof of Lemma 3. We can now present

Proof of Theorem 5: Suppose to the contrary that there exists an optimal program $\{x(t), y(t)\}$ with an associated value-loss sequence $\{\delta(t)\}$ that is not a Stiglitz' program. Let $\left\{x^{s}(t), y^{s}(t)\right\}$ be a Stiglitz program starting from $x(0)$ and with an associated value-loss sequence $\left\{\delta^{s}(t)\right\}$. An appeal to Lemma 4 and to Proposition 6 yields for all $T \in \mathbb{N}_{+}$,

$$
\begin{aligned}
\sum_{t=0}^{T}\left(b y(t)-b y^{s}(t)\right) & =\hat{p}\left(x^{s}(T+1)-x(T+1)\right)+\sum_{t=0}^{T} \delta^{s}(t)-\sum_{t=0}^{T} \delta(t) \\
& <\hat{p}\left(x^{s}(T+1)-x(T+1)\right)-\varepsilon
\end{aligned}
$$

Using Proposition 11, we can assert that $\limsup _{T \rightarrow \infty} \sum_{t=0}^{T}\left(b y(t)-b y^{s}(t)\right) \leq(-\varepsilon)$. Thus, we obtain $\liminf _{T \rightarrow \infty} \sum_{t=0}^{T}\left(b y^{s}(t)-b y(t)\right)=-\limsup _{T \rightarrow \infty} \sum_{t=0}^{T}\left(b y(t)-b y^{s}(t)\right) \geq \varepsilon$, a contradiction to the optimality of $\{x(t), y(t)\}$. This verifies the truth of the initial claim, and completes the argument that any optimal program is a Stiglitz program.

\footnotetext{
${ }^{48} \mathrm{~A}$ detailed verification of this claim would lead us outside the scope of an already long paper; see Khan-Mitra (2002) for details.
} 


\subsection{The Case of a General Felicity Function}

In the previous subsection, we considered optimal programs in the context of both the long and the short run when the felicity function is linear; which is to say a situation when a ceteris paribus transfer of a unit of consumption from a lower consumption level period to the higher one will definitely reduce social welfare. In the first subsection, we present a price-support property and some of its implications that have independent interest, and in a subsequent subsection, use these results to offer a sufficient condition under which an optimal program is a Stiglitz production program.

\subsubsection{The Price-Support Property and its Implications}

So far we have worked only with the golden-rule price system, and in this subsection we present McKenzie's price support property as Theorem 6 below. Since we do not exclude the situation where the economy has no stock of machines, $x_{o}=0$, the result is a direct consequence of methods available in McKenzie (1986; Proof of Lemma 1), rather than a corollary. We relegate to an Appendix the (straightforward) details of how McKenzie's interiority assumptions are fulfilled in our context, and allow his proof to work.

Theorem 6 Let $\{x(t), y(t)\}$ be an optimal program starting from an arbitrary initial stock, $x_{o} \in \mathbb{R}_{+}^{n}$. Then there exists a sequence $\{p(t)\}_{t=0}^{\infty}, p(t) \in \mathbb{R}_{+}^{n}$, such that for all $\left(x, x^{\prime}\right) \in \Omega$ and $y \in \Lambda\left(x, x^{\prime}\right)$,

$$
w(b y(t))+p(t+1) x(t+1)-p(t) x(t) \geq w(b y)+p(t+1) x^{\prime}-p(t) x .
$$

Next, or the convenience of the reader, we simply state in words the consequences of Theorem 6 , leaving their precise statement and proof to the Appendix. We can show that along an optimal program, investment in the machine of type $\sigma$ never ceases (Proposition 12), that there is investment in machines of this type only if they are valuable today (Proposition 14), and as a consequence, they are always valuable (Proposition 15), that machines types which are valuable today were valuable in the past ((Proposition 13) and that the prices are bounded (Proposition 17). We can use these results to establish expressions for the relative prices of produced machines (Proposition 18), and under the sufficient condition to be discussed below, an expression for the evolution of relative prices (Proposition 19). These results do not rely on linearity or strict concavity of the felicity function, and simply exploit the average-turnpike property of good programs, and as such rely on the uniqueness of the golden-rule stock. Thus the standing hypothesis presented as (1) above continues to be the driving force.

\subsubsection{A Sufficient Condition}

The scenario in which there can be a difference in the choice of techniques is one where the shortrun consumption requirements are quite different from the long-run consumption requirements on an optimal program. As we have seen in Section 5, the unique golden-rule type of machine, $\sigma$, is the best machine to use for meeting the long-run consumption requirements, regardless of whether the social welfare function is linear or strictly concave. In the short-run, however, the important question is which 
machine built today will provide the most consumption tomorrow, given an available amount of labor for new machine production today, and without taking into account the fact that the machine depreciates. This is clearly qualitatively different from the long-run (golden-rule) problem and points to $b_{i} / a_{i}$ rather than to $b_{i} /\left(1+d a_{i}\right)$. When the orderings of these two magnitudes differ, as they do in the example of Section 6.4, one machine is best for the short-run problem and another machine is best for the longrun problem. This seems to suggest that if the orderings coincide, then the machine that is best for the long-run remains best for the short-run, and only the golden-rule machine is produced and used. We assume that a unique type of machine $\sigma$ is best irrespective of the time horizon under which the planning exercise is being conducted, and furthermore, that it requires for its production more labor than a machine of any other type.

Assumption $1\left(b_{\sigma} / a_{\sigma}\right)>\max _{i \neq \sigma}\left(b_{i} / a_{i}\right)$ and $a_{\sigma}>\max _{i \neq \sigma} a_{i}$.

We can now show that under this congruence, the golden-rule machine $\sigma$ is the only type that is produced.

Theorem 7 Under Assumption 1, an optimal program is a Stiglitz production program.

The proof of Theorem 7 is relegated to the Appendix; while the basic intuition is clear, it requires the use of all of the consequences of the price-support property that we mentioned above, and which constitute Propositions 12-19 of the Appendix.

\section{Concluding Remarks}

If we leave aside the methodological reformulation of the RSS model in the vocabulary of the GaleMcKenzie reduced form, we see the principal contribution of this work: a complete resolution of the choice of technique problem in the long-run, and the identification and formalization of the Stiglitz policy as a cornerstone for the theory of transition dynamics. As regards the latter, three simple examples are of decisive importance, and they may also be of independent interest for future investigations of related issues that remain open. In conclusion, we briefly mention four of these.

Throughout this paper, we have emphasized the sharp and surprising differences that arise between our results and those of Stiglitz: in particular, the parameters $\xi_{i}$ do not appear in his paper. It is of some importance to settle the issue as to whether this is a consequence of the different treatment of time in the two papers, discrete versus continuous, or to the methods that Stiglitz had to work with in $1968 .^{49}$

In his retrospective, Stiglitz (1990, p. 61) observes the "greatest challenge facing growth theory":

We now need to understand better the relationship between the short-run behavior of the economy - in which imperfect information and imperfect competition in financial, labor, and product markets will play a central role - and its long-run dynamics.

\footnotetext{
${ }^{49}$ See Footnotes 9 and 40 above, and for preliminary work on this question, Khan-Mitra (2003).
} 
It is interesting that this remains a challenge even for a planning framework without uncertainty and the stark simplicity of the specifications of the RSS model, technological and otherwise. The complete characterization of the optimal path in the short-run remains an open problem when the planners' felicity function is linear but $\xi_{\sigma}<-1$, and when it is strictly concave.

We have drawn attention to the conceptual similarities between our work and that of Mitra-Wan (1987) on the economics of forestry. ${ }^{50}$ It would be of interest if the analogy is analytically explored in a synthesis based on the multi-sectoral setting of Koopmans (1971) and Koopmans-Hansen (1972). This work also gives a singular prominence to Kuhn-Tucker theory.

Finally, the results reported in this paper are a testimony to the strength of the standing hypothesis that there is a unique type of machine that minimizes effective labor costs and simultaneously maximizes the steady state consumption; see (1) above. It would be of interest to examine how the results are modified without this hypothesis.

\section{Appendix}

We begin with the proofs of three propositions in Section 4 .

Proof of Proposition 5: For any program $\{x(t), y(t)\}$, Proposition 1, and the fact that $y(t) \leq x(t)$ for all $t \in \mathbb{N}$, guarantee that the sequence $\{\bar{x}(T), \bar{y}(T)\}$ has at least one convergent subsequence. Let $\left(x^{\infty}, y^{\infty}\right)$ be the limit of one such subsequence. We leave it to the reader to check that $\left(x^{\infty}, x^{\infty}\right) \in \Omega$ and that $y^{\infty} \in \Lambda\left(x^{\infty}, x^{\infty}\right)$. Since $\{x(t), y(t)\}$ is a good program, there exists $G \in \mathbb{R}$ such that for all $T \in \mathbb{N}_{+}$,

$$
G / T \leq(1 / T) \sum_{t=0}^{T-1}(w(b y(t))-w(b \hat{y})) \leq w(b \bar{y}(T))-w(b \hat{y})
$$

where the second assertion is a consequence of the concavity of $w(\cdot)$. On taking limits as $T \rightarrow \infty$ along the chosen subsequence, and on appealing to the continuity of $w$, we obtain $w\left(b y^{\infty}\right) \geq w(b \hat{y})$. Hence $x^{\infty}$ is a golden-rule stock. Since the golden rule stock is unique, $x^{\infty}=\hat{x}$, and since $w$ is strictly increasing, $y^{\infty}=\hat{y}$. Since we worked with an arbitrary convergent subsequence, the argument is complete.

Proof of Proposition 8: Proposition 2 guarantees that there exists a good program $\{x(t), y(t)\}$ from $x_{o}$, and hence from Proposition 6, we obtain that $\Delta\left(x_{o}\right)<\infty$. Since $\{\delta(t)\}$ is a non-negative sequence, certainly $0 \leq \Delta\left(x_{o}\right)$.

For any $\nu \in \mathbb{N}_{+}$, there exists a program $\left\{x^{\nu}(t), y^{\nu}(t)\right\}$ from $x_{o}$ such that its associated value-loss sequence $\left\{\delta^{\nu}(t)\right\}$ satisfies

$$
\sum_{t=0}^{\infty} \delta^{\nu}(t) \leq \Delta\left(x_{o}\right)+(1 / \nu)
$$

From Proposition 1, the sequence $\left\{x^{\nu}(t)\right\}_{\nu=1}^{\infty}$ is bounded independently of $\nu$ (and of $t$ but not of $x_{o}$ ). Since $y^{\nu}(t) \leq x^{\nu}(t)$, the same is true of the sequence $\left\{y^{m}(t)\right\}_{m=1}^{\infty}$. We can now appeal to a diagonalization argument (see Rudin (1967; Theorem 7.23) to extract a subsequence indexed by $\nu_{k}, k \in \mathbb{N}_{+}$, that converges for all $t \in \mathbb{N}$. Let $\left\{x^{\prime}(t), y^{\prime}(t)\right\}$ be the limit of this subsequence. Let $\delta^{\prime}(t)=\hat{p}\left(x^{\prime}(t)-x^{\prime}(t+1)\right)-\left(w\left(b y^{\prime}(t)\right)-w(b \hat{y})\right)$ for all $t \in \mathbb{N}$. Since $w(\cdot)$ is a continuous function, $\delta^{\nu_{k}}(t) \longrightarrow \delta^{\prime}(t)$ for all $t \in \mathbb{N}$.

It is easy to check that $\left\{x^{\prime}(t), y^{\prime}(t)\right\}$ is a program from $x_{0}$. Since $\left\{\delta^{\prime}(t)\right\}$ is its associated value-loss sequence, certainly $\sum_{t=0}^{\infty} \delta^{\prime}(t) \geq \Delta\left(x_{o}\right)$. If we now consider the set of natural numbers $\mathbb{N}$ as a measure space

\footnotetext{
${ }^{50}$ See Footnotes 3 and 21.
} 
equipped with a counting measure, we can appeal to Fatou's lemma (see Rudin (1967; Theorem 11.31) via (8) to assert

$$
\Delta\left(x_{o}\right) \geq \lim _{k \rightarrow \infty} \sum_{t=0}^{\infty} \delta^{\nu_{k}}(t) \geq \sum_{t=0}^{\infty} \liminf _{k \rightarrow \infty} \delta^{\nu_{k}}(t)=\sum_{t=0}^{\infty} \delta^{\prime}(t) .
$$

This completes the proof of our claim. ${ }^{51}$

Proof of Proposition 9: We know from Proposition 7 that the program $\{x(t), y(t)\}$ is good. Now suppose that it is not optimal. Then there exists a program $\left\{x^{\prime}(t), y^{\prime}(t)\right\}, x^{\prime}(0)=x(0)$, a number $\varepsilon>0$ and a time period $t_{\varepsilon}$ such that $\sum_{t=1}^{T}\left[w\left(b y^{\prime}(t)\right)-w(b y(t))\right]>\varepsilon$ for all $T \geq t_{\varepsilon}$. Since $\{x(t), y(t)\}$ is good, $\left\{x^{\prime}(t), y^{\prime}(t)\right\}$ is good, and by Proposition 5 both programs satisfy the average turnpike property. Let $\left\{\delta^{\prime}(t)\right\}$ be the value-loss sequence associated with the latter program. We can now appeal to Proposition 6 to assert that for all $T \geq t_{\varepsilon}$,

$$
\varepsilon<\sum_{t=0}^{T}\left(w\left(b y^{\prime}(t)\right)-w(b y(t))\right)=\hat{p}\left(x(T+1)-x^{\prime}(T+1)\right)+\sum_{t=0}^{T} \delta(t)-\sum_{t=0}^{T} \delta^{\prime}(t) .
$$

From the minimality of $\sum_{t=0}^{\infty} \delta(t)$, there exists $t_{\varepsilon}^{\prime}$ such that for all $T \geq t_{\varepsilon}^{\prime},(\varepsilon / 2)<\hat{p}\left(\bar{x}(T+1)-\bar{x}^{\prime}(T+1)\right)=$ $\hat{p}\left(\bar{x}(T+1)-\hat{x}+\hat{x}-\bar{x}^{\prime}(T+1)\right)$. On taking limits with respect to $T$, we obtain a contradiction.

Next, we turn to the Example in Subsection 6.4

Computations for the example in Subsection 6.4: First we define a program $\{x(t), z(t)\}$ from $x_{o}$ which reaches the golden-rule stock in a finite number of periods.

$$
\begin{aligned}
& x(0)=(0.5,0)=y(0), a z(1)=(0.2+0.3), z(1)=(0.1,0.1) \\
& x(1)=(0.375,0.1)=y(1), a z(2)=(0.09+0.435), z(2)=(0.045,0.145) ; \\
& x(2)=(0.25125,0.2) \equiv(\alpha, 0.2)=y(2), a z(3)=(0.54), z(3)=(0,0.18) ; \\
& x(3)=(m \alpha, 0.29), y(3)=(0.1375,0.29), a z(4)=(0.57), z(4)=(0,0.19) ; \\
& x(4)=\left(m \alpha^{2}, 0.3495\right), y(4)=(0.075,0.34), a z(5)=(0.57), z(4)=(0,0.19) ; \\
& x(5)=\left(m \alpha^{3}, 0.382225\right), y(5)=(0.04,0.38), a z(6)=(0.579), z(6)=(0,0.193) ; \\
& x(6)=\left(m \alpha^{4}, 0.40322375\right)=\left(m \alpha^{4}, b\right), y(6)=(0,0.4), a z(7)=(0.6), z(7)=(0,0.2) ; \\
& x(7)=\left(m \alpha^{5}, m b+0.2\right), y(7)=(0,0.4), a z(8)=(0.6), z(8)=(0,0.2) ; \\
& x(8)=\left(m \alpha^{6}, 1 / 2.35\right) \equiv\left(m \alpha^{6}, \beta\right), y(8)=(0, \beta), a z(9)=(1-\beta), z(9)=(0,0.45 \beta) ;
\end{aligned}
$$

and for all $t \geq 9$,

$$
x(t)=\left(m \alpha^{t-2}, 1 / 2.35\right), y(t)=(0,1 / 2.35), a z(t+1)=(1.35 / 2.35), z(t+1)=(0,0.45 / 2.35) .
$$

It can be checked (although it is tedious to do so) that the above sequence defines a program from $x_{o}$. Note that for all $t \in \mathbb{N}$, the consumption in period $(t+1)$ is given $b y(t)$. Hence $b y(0)=2, b y(1)=2, b y(2)=$ $2.005, b y(3)=2, b y(4)=2, b y(5)=2.06, b y(6)=2, b y(7)=2, b y(t)=5 / 2.35 \approx 2.1276$ for all $t \geq 8$. Thus, the utility along this program is non-negative at all dates, and $u(x(t), x(t+1))=u(\hat{x}, \hat{x})>0$ for all $t \geq 8$. Using this, we have for all $T \geq 8$,

$$
\sum_{t=0}^{T}[u(x(t), x(t+1))-u(\hat{x}, \hat{x})] \geq-8 u(\hat{x}, \hat{x}) \geq-8 .
$$

Now, consider the optimal program $\left\{x^{\prime}(t), x^{\prime}(t+1)\right\}$ from $x_{o}$, and suppose that machines of type 1 are not produced at all in period 1 ; that is, $z_{1}^{\prime}(1)=0$. Denote $y_{1}^{\prime}(0)$ by $\lambda$, and note that $\lambda \leq 0.5$. We split up our analysis into two cases: (i) $\lambda \leq 0.49$; (ii) $\lambda>0.49$.

\footnotetext{
${ }^{51}$ For a direct argument that does not appeal to integration and to Fatou's lemma, see Brock (1970, p. 278).
} 
In case $(\mathrm{i}), c^{\prime}(1)=b y^{\prime}(0)=4 \lambda \leq 1.96$, so that $u\left(x^{\prime}(0), x^{\prime}(1)\right) \leq-40$. We also have $a z^{\prime}(1) \leq(0,1)$, and $z^{\prime}(1) \leq(0,1 / 3)$. Thus, $y^{\prime}(1) \leq x^{\prime}(1) \leq(0.275,1 / 3)$, and $c(1)=b y^{\prime}(1)<3$, so that $u\left(x^{\prime}(1), x^{\prime}(2)\right)<1$. Thus, in this case, we have:

$$
\sum_{t=0}^{1}\left[u\left(x^{\prime}(t), x^{\prime}(t+1)\right)-u(\hat{x}, \hat{x})\right] \leq-39 .
$$

In case (ii), $c^{\prime}(1)=b y^{\prime}(0) \leq 4(0.5)=2$, so that $u\left(x^{\prime}(0), x^{\prime}(1)\right) \leq 0$. We also have $a z^{\prime}(1) \leq(0,0.51)$, and so $z^{\prime}(1) \leq(0,0.17)$. Thus, $y^{\prime}(1) \leq x^{\prime}(1) \leq(0.275,0.17)$, and $c^{\prime}(1)=b y^{\prime}(1) \leq 1.95$, so that $u\left(x^{\prime}(1), x^{\prime}(2)\right) \leq-50$. Thus, in this case, we have:

$$
\sum_{t=0}^{1}\left[u\left(x^{\prime}(t), x^{\prime}(t+1)\right)-u(\hat{x}, \hat{x})\right] \leq-50 .
$$

We now obtain a bound for the sum of utilities (minus the golden rule utility) on this program from time period 2 onwards. Towards this end, appeal to Lemma 1 to obtain for $T \geq 2$,

$$
\sum_{t=2}^{T}\left[u\left(x^{\prime}(t), x^{\prime}(t+1)\right)-u(\hat{x}, \hat{x})\right] \leq \hat{p} x^{\prime}(2)+\hat{p} \hat{x} .
$$

From the labor constraint, we know that $a x^{\prime}(2) \leq 1+(1-d) a x^{\prime}(1)$, and in both cases (i) and (ii), we have $\left(1+(1-d) a x^{\prime}(1)\right) \leq 1.8525<2$, so that $a x^{\prime}(2)<2$, and $x^{\prime}(2) \leq(1,2 / 3)$. Also, $\hat{p} \leq(5,7)$, so $\hat{p} x^{\prime} \leq 10$, and $\hat{p} \hat{x} \leq 3$. Using this in (12), we obtain

$$
\sum_{t=2}^{T}\left[u\left(x^{\prime}(t), x^{\prime}(t+1)\right)-u(\hat{x}, \hat{x})\right] \leq 13 .
$$

Using (10) and (13) in case (i), and (11) and (13) in case (ii), we obtain in either case for $T \geq 2$

$$
\sum_{t=0}^{T}\left[u\left(x^{\prime}(t), x^{\prime}(t+1)\right)-u(\hat{x}, \hat{x})\right] \leq-26 .
$$

Using (9) and (14), we get for $T \geq 8$ (in both cases):

$$
\sum_{t=0}^{T}\left[u(x(t), x(t+1))-u\left(x^{\prime}(t), x^{\prime}(t+1)\right)\right] \geq 18,
$$

which contradicts the optimality of $\left\{x^{\prime}(t), y^{\prime}(t)\right\}$ from $x_{o}$. This establishes that $z_{1}^{\prime}(1)>0$; that is, some machines of type 1 are produced in period 1 along the optimal program.

Next, we turn to the results in Subsection 7.1.

Proof of Lemma 3: Let $\left\{x^{s}(t), y^{s}(t)\right\}$ be a Stiglitz program with an associated gross investment sequence $\left\{z^{s}(t+1)\right\}$ and an associated value-loss sequence $\left\{\delta^{s}(t)\right\}$. We shall denote corresponding values of any other (candidate) program starting from $x^{s}(0)$ by $\{x(t), y(t)\},\{z(t+1)\}$, and $\{\delta(t)\}$. We shall consider three different ranges for the value of $\xi_{\sigma}$ and make repeated use of (7) and of Definition 8.

Case (i) $0<\xi_{\sigma}<1$ :

Suppose that for any $t \in \mathbb{N}, 0 \leq \sum_{i \in D} x_{i}^{s}(t) \leq 1$. In this case, we see from (i) of Definition 8 that $y_{i}^{s}(t)=x_{i}^{s}(t)$ for all $i \in D, y_{i}^{s}(t)=0$ for all $i \notin D$, and $z^{s}(t+1)=\left(1 / a_{\sigma}\right)\left(1-\sum_{i \in D} x_{i}^{s}(t)\right) e(\sigma)$. We leave it to the reader to check that $\left(x^{s}(t), x^{s}(t+1)\right) \in \Omega$ and that $y^{s}(t) \in \Lambda\left(x^{s}(t), x^{s}(t+1)\right)$. On substituting these values in (7), we obtain that ${ }^{52}$

$$
\delta^{s}(t)=\sum_{i \in D}\left(c_{\sigma}-b_{i}\right) x_{i}^{s}(t)+d q x^{s}(t)=\sum_{i \in D}\left(c_{\sigma}-b_{i}+d q_{i}\right) x_{i}^{s}(t)+d \sum_{i \notin D} q_{i} x_{i}^{s}(t)
$$

\footnotetext{
${ }^{52}$ Note that in the third equality we use the identity referred to in Footnote 35 above. We shall not draw attention to this in the sequel.
} 


$$
=\sum_{i \in D}\left(c_{\sigma}-c_{i}\right) x_{i}^{s}(t)+d \sum_{i \notin D} q_{i} x_{i}^{s}(t) .
$$

Again from (7) we obtain ${ }^{53}$

$$
\begin{aligned}
\delta(t) & \geq \sum_{i \in D}\left(c_{\sigma}-b_{i}\right) y_{i}(t)+d q x(t) \geq \sum_{i \in D}\left(c_{\sigma}-b_{i}\right) x_{i}(t)+d q x(t) \\
& =\sum_{i \in D}\left(c_{\sigma}-b_{i}+d q_{i}\right) x_{i}(t)+d \sum_{i \notin D} q_{i} x_{i}(t)=\sum_{i \in D}\left(c_{\sigma}-c_{i}\right) x_{i}(t)+d \sum_{i \notin D} q_{i} x_{i}(t) .
\end{aligned}
$$

Next, we claim that for all $t \in \mathbb{N}, x_{i}(t) \geq x_{i}^{s}(t)$ for all $i \neq \sigma$. Since the candidate program starts from the same initial stock as the Stiglitz program, the claim holds for $t=0$. Suppose it to be true for any $t \in \mathbb{N}$, in keeping with the induction hypothesis. Then

$$
x_{i}^{s}(t+1)=(1-d) x_{i}^{s}(t) \leq(1-d) x_{i}(t) \leq(1-d) x_{i}(t)+z_{i}(t+1)=x_{i}(t+1) .
$$

Given the standing hypothesis, it is clear that for all $t \in \mathbb{N}, \delta^{s}(t) \leq \delta(t)$. Thus, we need only to verify that the Stiglitz program is feasible in the sense that once in the range $0 \leq \sum_{i \in D} x_{i}^{s}(t) \leq 1$, the program always remains in it. We proceed by induction. For any $t \in \mathbb{N}$, note that

$$
x_{i}^{s}(t+1)= \begin{cases}(1-d) x_{i}^{s}(t) & \text { for all } i \neq \sigma \\ (1-d) x_{\sigma}^{s}(t)+\left(1 / a_{\sigma}\right)\left(1-\sum_{i \in D} x_{i}^{s}(t)\right) & \text { for } i=\sigma\end{cases}
$$

Since $0 \leq \sum_{i \in D} x_{i}^{s}(t) \leq 1$, we obtain from (15) that $z_{\sigma}(t+1)=x_{\sigma}^{s}(t+1)-(1-d) x_{\sigma}^{s}(t) \geq 0$, and that

$$
\begin{aligned}
\sum_{i \in D} x_{i}^{s}(t+1) & =(1-d) \sum_{i \in D} x_{i}^{s}(t)+\frac{1}{a_{\sigma}}\left(1-\sum_{i \in D} x_{i}^{s}(t)\right) \\
& =\left(1-d-\frac{1}{a_{\sigma}}\right) \sum_{i \in D} x_{i}^{s}(t)+\frac{1}{a_{\sigma}}=\xi_{\sigma} \sum_{i \in D} x_{i}^{s}(t)+\frac{1}{a_{\sigma}}
\end{aligned}
$$

Given the possible values of $\xi_{\sigma}$, we obtain $0<\sum_{i \in D} x_{i}^{s}(t+1)<1$.

We can now collect these steps to assert that for all $t \in \mathbb{N}, \delta^{s}(t) \leq \delta(t)$, and hence that $\sum_{t=0}^{\infty} \delta^{s}(t)=$ $\Delta\left(x^{s}(0)\right)$.

Next we turn to the case when for any $t \in \mathbb{N}, \sum_{i \in D} x_{i}^{s}(t)>1, x_{1}^{s}(t)<1$. In this case, we see from (iii) of Definition 8 that $y_{i}^{s}(t)=x_{i}^{s}(t)$ for all $i<i_{o}, y_{i_{o}}^{s}(t)=1-\sum_{i=1}^{i_{o}-1} x_{i}^{s}(t), y_{i}^{s}(t)=0$ for all $i>i_{o}$, and that $z_{i}^{s}(t+1)=0$ for all $i$. We leave it to the reader to check that $\left(x^{s}(t), x^{s}(t+1)\right) \in \Omega$ and that $y^{s}(t) \in \Lambda\left(x^{s}(t), x^{s}(t+1)\right)$. On substituting these values in (7), we obtain that

$$
\begin{aligned}
\delta^{s}(t) & =\sum_{i=1}^{i_{o}-1}\left(c_{\sigma}-b_{i}\right) x_{i}^{s}(t)+\left(c_{\sigma}-b_{i_{o}}\right)\left(1-\sum_{i=1}^{i_{o}-1} x_{i}^{s}(t)\right)+d q x^{s}(t) \\
& =\sum_{i=1}^{i_{o}-1}\left(c_{\sigma}-c_{i}\right) x_{i}^{s}(t)+\left(c_{\sigma}-b_{i_{o}}\right)\left(1-\sum_{i=1}^{i_{o}-1} x_{i}^{s}(t)\right)+d \sum_{i \geq i_{o}} q_{i} x_{i}^{s}(t),
\end{aligned}
$$

and for any other (candidate) program with $D_{o}=D /\left\{1, \cdots, i_{o}\right\}$ that

$$
\begin{aligned}
\delta(t) & \geq \sum_{i=1}^{i_{o}-1}\left(c_{\sigma}-b_{i}\right) y_{i}(t)+\left(c_{\sigma}-b_{i_{o}}\right) y_{i_{o}}(t)+\sum_{i \in D_{o}}\left(c_{\sigma}-b_{i}\right) y_{i}(t)+d q x(t) \\
& \geq \sum_{i=1}^{i_{o}-1}\left(c_{\sigma}-b_{i}\right) x_{i}(t)+\left(c_{\sigma}-b_{i_{o}}\right) y_{i_{o}}(t)+\sum_{i \in D_{o}}\left(c_{\sigma}-b_{i}\right) y_{i}(t)+d q x(t) \\
& =\sum_{i=1}^{i_{o}-1}\left(c_{\sigma}-c_{i}\right) x_{i}(t)+\left(c_{\sigma}-b_{i_{o}}\right) y_{i_{o}}(t)+\sum_{i \in D_{o}}\left(c_{\sigma}-b_{i}\right) y_{i}(t)+d \sum_{i \geq i_{o}} q_{i} x_{i}(t) .
\end{aligned}
$$

\footnotetext{
${ }^{53}$ We rely on the standing hypothesis (1) and on the definition of desirable machines, in addition to the feasibility of the program.
} 
Now by hypothesis, for all $i \in D_{o}, b_{i} \leq b_{i_{o}}$ so that: $\sum_{i \in D_{o}}\left(c_{\sigma}-b_{i}\right) y_{i}(t) \geq\left(c_{\sigma}-b_{i_{o}}\right) \sum_{i \in D_{o}} y_{i}(t)$. Hence we obtain

$$
\left.\left(c_{\sigma}-b_{i_{o}}\right) y_{i_{o}}(t)+\sum_{i \in D_{o}}\left(c_{\sigma}-b_{i}\right) y_{i}(t)\right) \geq\left(c_{\sigma}-b_{i_{o}}\right) \sum_{i \in D_{o} \cup i_{o}} y_{i}(t) \geq\left(c_{\sigma}-b_{i_{0}}\right) \sum_{i \in D_{o} \cup i_{o}} x_{i}(t) .
$$

Also, by definition of $\left\{x^{s}(t), y^{s}(t)\right\}$, we have $\sum_{i \in D_{o} \cup i_{o}} x_{i}(t) \geq \sum_{i \in D_{o} \cup i_{o}} x_{i}^{s}(t) \geq\left(1-\sum_{i=1}^{i_{o}-1} x_{i}^{s}(t)\right)$. Now $\sum_{i \in D} x_{i}^{s}(0)>1$ implies that there exists a first $t_{1} \in T$ such that $\sum_{i \in D} x_{i}^{s}\left(t_{1}\right) \leq 1$. For the Stiglitz program, we know that for all $t \in \mathbb{N}, t<t_{1}, z_{i}^{s}(t+1)=0$ for all $i$, and hence that $x^{s}(t) \leq x(t)$. In particular, $x_{1}^{s}(t)<1$ for all $t \in \mathbb{N}, t<t_{1}$. This implies that for all $t \in \mathbb{N}, t<t_{1}, \delta^{s}(t) \leq \delta(t)$. [Note that $i_{o}$ may vary with $t$, but given our period-by-period verification, it is of no consequence.] But for $t \geq t_{1}$, we are in the case considered earlier, and hence we can assert that for all $t \in \mathbb{N}, \delta^{s}(t) \leq \delta(t)$, and hence that $\sum_{t=0}^{\infty} \delta^{s}(t)=\Delta\left(x^{s}(0)\right)$.

Next we turn to the case when for any $t \in \mathbb{N}, \sum_{i \in D} x_{i}^{s}(t)>1, x_{1}^{s}(t)>1$. In this case, we see from (ii) of Definition 8 that $y_{1}^{s}(t)=1, y_{i}^{s}(t)=0$ for all $i \neq 1$, and that $z_{i}^{s}(t+1)=0$ for all $i$. We leave it to the reader to check that $\left(x^{s}(t), x^{s}(t+1)\right) \in \Omega$ and that $y^{s}(t) \in \Lambda\left(x^{s}(t), x^{s}(t+1)\right)$. On substituting these values in (7), we obtain that $\delta^{s}(t)=\left(c_{\sigma}-b_{1}\right)+d q x^{s}(t)$, and for any other (candidate) program that $\delta(t) \geq \sum_{i \in D}\left(c_{\sigma}-b_{i}\right) y_{i}(t)+d q x(t)$. Now by hypothesis, $b_{i} \leq b_{1}$, so that: $\sum_{i \in D}\left(c_{\sigma}-b_{i}\right) y_{i}(t) \geq\left(c_{\sigma}-b_{1}\right) \sum_{i \in D} y_{i}(t)$. Since $\sum_{i \in D} y_{i}(t) \leq 1$ by definition of a program, and since $\left(c_{\sigma}-b_{1}\right) \leq 0, \sum_{i \in D}\left(c_{\sigma}-b_{i}\right) y_{i}(t) \geq\left(c_{\sigma}-b_{1}\right)$. Since both programs start from the same initial stock and $x_{1}^{s}(t)>1$ implies $x_{i}^{s}(t-r)>1$ for all $r=0, \cdots, t-1, x_{i}(t) \geq x_{i}^{s}(t)$ for all $i$. Hence $\delta^{s}(t) \leq \delta(t)$.

It is clear that if $x_{1}^{s}(0)>1$, there exists $t_{1} \in T$ such that $x_{1}^{s}\left(t_{1}\right) \leq 1$. We have already seen that $\delta^{s}(t) \leq \delta(t)$ for all $t<t_{1}$. Now either $\sum_{i \in D} x_{i}^{s}\left(t_{1}\right) \leq 1$, in which case we appeal to the first case, or $\sum_{i \in D} x_{i}^{s}\left(t_{1}\right)>1$, in which case we appeal to the second case and complete the demonstration that for all $t \in \mathbb{N}, \delta^{s}(t) \leq \delta(t)$. Hence $\sum_{t=0}^{\infty} \delta^{s}(t)=\Delta\left(x^{s}(0)\right)$.

Case (ii) $0>\xi_{\sigma} \geq-1$ :

Suppose that for any $t \in \mathbb{N},(1-d) \leq \sum_{i \in D} x_{i}^{s}(t) \leq 1$. On examining the argument for this subcase within case (i) above, we see that the value of $\xi_{\sigma}$ is used only to verify the feasibility of the Stiglitz program in equation (16). However, with $0>\xi_{\sigma} \geq-1, \sum_{i \in D} x_{i}^{s}(t) \leq 1$ implies $\xi_{\sigma} \sum_{i \in D} x_{i}^{s}(t) \geq \xi_{\sigma}$, and therefore

$$
\sum_{i \in D} x_{i}^{s}(t+1)=\xi_{\sigma} \sum_{i \in D} x_{i}^{s}(t)+\left(1 / a_{\sigma}\right) \geq \xi_{\sigma}+\left(1 / a_{\sigma}\right)=(1-d) .
$$

Furthermore, $\sum_{i \in D} x_{i}^{s}(t) \geq(1-d)$ implies $\xi_{\sigma} \sum_{i \in D} x_{i}^{s}(t) \leq \xi_{\sigma}(1-d)$, and therefore

$$
\sum_{i \in D} x_{i}^{s}(t+1)=\xi_{\sigma} \sum_{i \in D} x_{i}^{s}(t)+\left(1 / a_{\sigma}\right) \leq \xi_{\sigma}(1-d)+\left(1 / a_{\sigma}\right)=(1-d)^{2}+\left(d / a_{\sigma}\right)
$$

Since $\xi_{\sigma}=1-d-\left(1 / a_{\sigma}\right) \geq-1,\left(1 / a_{\sigma}\right) \leq 2-d$, which implies that $\left(d / a_{\sigma}\right) \leq(2-d) d$ and hence that $(1-d)^{2}+\left(d / a_{\sigma}\right) \leq(1-d)^{2}+(2-d) d=1$. We have thus shown that once in the range $(1-d) \leq \sum_{i \in D} x_{i}^{s}(t) \leq 1$, the program always remains in it.

For the other two subcases in the argument within case (i) above, we note that $\xi_{\sigma}$ plays no role and that everything hinges on the value of $d$. Thus the only remaining case to be considered is when $0 \leq \sum_{i \in D} x_{i}^{s}(t)<$ $(1-d)$. Here there are two possibilities: either $\sum_{i \in D} x_{i}^{s}(t+1) \leq 1$ or $\sum_{i \in D} x_{i}^{s}(t+1)>1$. Since we have already shown that $\sum_{i \in D} x_{i}^{s}(t+1) \geq(1-d)$, there is nothing further to be shown under the first possibility. Under the second, there exists a first $t_{1} \in \mathbb{N}, t_{1}>t$ such that $\sum_{i \in D} x_{i}^{s}\left(t_{1}\right) \leq 1$. Since $\sum_{i \in D} x_{i}^{s}\left(t_{1}\right)=$ $(1-d) \sum_{i \in D} x_{i}^{s}\left(t_{1}-1\right)>(1-d)$, we are in the case analyzed above, and the demonstration is complete.

Case (iii) $\xi_{\sigma}=0$ :

This is a trivial case where $\left(1 / a_{\sigma}\right)=1 /\left(1+d a_{\sigma}\right)=\hat{x}$. Suppose that for any $t \in \mathbb{N}, 0 \leq \sum_{i \in D} x_{i}^{s}(t) \leq 1$. Then we see from Equation (16) that $\sum_{i \in D} x_{i}^{s}(t)=1 / a_{\sigma}$. For the other subcases, the argument is identical to that presented under case (i).

We have now covered all possible cases, and the proof of the lemma is complete.

Proof of Proposition 11: We shall prove the proposition for an optimal program; identical computations hold for a Stiglitz program. By virtue of Theorem 3, we know that eventually machines of type other than $\sigma$ are 
never built in any optimal program, and hence for all $i \neq \sigma, \lim _{t \rightarrow \infty} x_{i}(t)=0$. Since $y_{i}(t) \leq x_{i}(t)$, the proof of the first claim is complete. In the case of machines of type $\sigma$, we appeal to Lemma 2 to characterize the behavior of paths on the von Neumann facet. A subsequent appeal to Theorem 3 then proves the second claim. Towards the first requirement, note that

$$
\begin{aligned}
x_{\sigma}(t+1) & =(1-d) x_{\sigma}(t)+z_{\sigma}(t+1)=(1-d) x_{\sigma}(t)+\left(1 / a_{\sigma}\right)\left(1-\sum_{i \in D} x_{i}(t)\right) \\
& =\xi_{\sigma} x_{\sigma}(t)-\left(1 / a_{\sigma}\right)\left(1-\sum_{i \in D /\{\sigma\}} x_{i}(t)\right)=\xi_{\sigma} x_{\sigma}(t)-\left(1 / a_{\sigma}\right)\left(1-(1-d)^{t} \sum_{i \in D /\{\sigma\}} x_{i}(0)\right) .
\end{aligned}
$$

Let $g(t)$ denote $\left(1 / a_{\sigma}\right)\left(1-(1-d)^{t} \sum_{i \in D /\{\sigma\}} x_{i}(0)\right)$. We can then appeal to Elaydi (1999; p.4) to obtain

$$
\begin{aligned}
x_{\sigma}(t) & =\xi_{\sigma}^{t} x_{\sigma}(0)+\sum_{\tau=0}^{t-1} \xi_{\sigma}^{t-\tau-1} g(\tau)=\xi_{\sigma}^{t} x_{\sigma}(0)+\left(1 / a_{\sigma}\right) \sum_{\tau=0}^{t-1} \xi_{\sigma}^{t-\tau-1}\left(1-(1-d)^{\tau} \sum_{i \in D / \sigma} x_{i}(0)\right) \\
& \left.=\left(1 / a_{\sigma}\right) \sum_{\tau=0}^{t-1} \xi_{\sigma}^{\tau}+\xi_{\sigma}^{t} x_{\sigma}(0)-\left(1 / a_{\sigma}\right) \sum_{\tau=0}^{t-1} \xi_{\sigma}^{t-\tau-1}(1-d)^{\tau} \sum_{i \in D /\{\sigma\}} x_{i}(0)\right) .
\end{aligned}
$$

Since $-1<\xi_{\sigma}<1$, this yields $\lim _{t \rightarrow \infty} x_{\sigma}(t)=1 /\left(1+d a_{\sigma}\right)$, and hence that $\lim _{t \rightarrow \infty} y_{\sigma}(t)=1 /\left(1+d a_{\sigma}\right)$ and $\lim _{t \rightarrow \infty} z_{\sigma}(t)=d a_{\sigma} /\left(1+d a_{\sigma}\right)$.

Proof of Lemma 4: Since the program $\{(x(t), y(t)\}$ starts from the same initial stock as a Stiglitz program, and there exists a first time period $t_{1} \in \mathbb{N}$, at which it departs from the Stiglitz program, we can appeal to the computations of $\delta\left(t_{1}\right)$ and $\delta^{s}\left(t_{1}\right)$ in each of the relevant cases delineated in the proof of Lemma 3 to show that they will hold with strict inequality. This furnishes a proof of the claim.

Proof of Theorem 6: The result is (part of) the assertion of Lemma 1 of McKenzie (1986). McKenzie works with a general setting in which the transition possibility set and the reduced form utility function are allowed to change over time. He utilizes assumptions I, II and III to derive his assertions. Assumption I is a requirement that $u(\cdot, \cdot)$ is concave and closed and that $\Omega$ is convex. Assumption II is the requirement that there exists $\zeta_{o} \in \mathbb{R}_{+}$such that for all $\left(x, x^{\prime}\right) \in \Omega, x<\zeta_{o}$, there exists $\zeta \in \mathbb{R}_{+}$such that $x^{\prime}<\zeta$. The specifications on $u$ and on $\Omega$ presented in Section 2.4 and Proposition 1 above guarantee that I and II are fulfilled in our setting.

Assumption III is also a hypothesis on what McKenzie terms the "maximal path of capital accumulation" and on what we are referring to here as an optimal program. He works with the sets $P_{t}$ and $K_{t}$ for all $t \in \mathbb{I N}$. In our stationary setting these sets are independent of $t$. $P_{t}$ concerns only $\Omega$ and in our setting, it is $\mathbb{R}_{+}^{n}$ for all $t \in \mathbb{I N}$. $K_{t}$ concerns also the optimal program, and since Theorem 2 above guarantees that there is an optimal program from any initial stock, it is also $\mathbb{R}_{+}^{n}$ for all $t \in \mathbb{N}$. Now Assumption III requires that for any optimal program $\{x(t), y(t)\}, x(t)$ is in the relative interior of $\mathbb{R}_{+}^{n}$ for all $t \in \mathbb{N}$. If $x(0)>0$, this assumption is fulfilled for any program, leave alone an optimal one. However, $x(0)=0$ is not in the relative interior of $\mathbb{R}_{+}^{n}$, and Assumption III is not fulfilled. The relevant observation here is to apply McKenzie's Lemma 1 for the case $x(0)>0$, and then to observe that for any optimal path starting from $0, u(0, x(1))=0$, and hence that $V(x(0))=V(x(1))$. (Note that McKenzie's $V\left(k_{t}\right)$ is independent of the value of the golden-rule stock and is defined solely with respect to the optimal program.) It is clear that $x(1)>0$ by the optimality of the program. The price-support for $V(x(1))$ also works for $V(x(0))$, and allows the induction to proceed. The demonstration is then complete.

We now turn towards the proof of Theorem 7, and develop the implications of the price-support property informally described in Subsection 7.2.1. In their precise statements to follow, Propositions 12-19, the hypotheses of Theorem 7 are in force. This is to say that $\{x(t), y(t)\}$ is an optimal program, and $\{p(t)\}$ its associated pricesupport.

Proposition 12 There exists a sequence $\left\{t_{i}\right\}_{i \in \mathbb{N}_{+}}$such that $z_{\sigma}\left(t_{i}\right)>0$ for all $i \in \mathbb{N}_{+}$.

Proof: Suppose this not to be the case. Then there exists $T \in \mathbb{N}$ such that for all $t \geq T, z_{\sigma}(t)=0$. Since all machines depreciate at the rate $d \in(0,1)$, this implies that $x_{\sigma}(t) \rightarrow 0$ as $t \rightarrow \infty$, and therefore that the time-average of $x_{\sigma}(t), \bar{x}_{\sigma}(t) \rightarrow 0$ as $t \rightarrow \infty$. An appeal to Propositions 5 and 10 furnishes a contradiction and completes the argument. 
Proposition 13 For any $t \in \mathbb{N}$, and any $i=1, \cdots, n,(i)(1-d) p_{i}(t+1) \leq p_{i}(t)$, and $(i i) p_{i}(t+1)>0 \Longrightarrow$ $p_{i}(t)>0$.

Proof: For any time-period $t$ and any machine of type $i$, let $x=x(t)+e(i) \varepsilon, z=z(t+1), x^{\prime}=(1-d) x+z, y=$ $y(t)$ where $\varepsilon>0$. Then, $\left(x, x^{\prime}\right) \in \Omega$, and $y \in \Lambda\left(x, x^{\prime}\right)$. Using Theorem 6 , we have $p_{i}(t+1)(1-d) \varepsilon-p_{i}(t) \varepsilon \leq 0$, which yields result (i). In particular, if for some $t \in \mathbb{N}$, and $i=(1, \cdots, n), p_{i}(t+1)>0$, then we must have $p_{i}(t)>0$.

Proposition 14 For any $t \in \mathbb{N}, z_{\sigma}(t+1)>0$ implies $p_{\sigma}(t)>0$.

Proof: Suppose that for some time-period $t, z_{\sigma}(t+1)>0$ and $p_{\sigma}(t)=0$. Then Proposition 13 implies that $p_{\sigma}(t+1)=0$. Pick $\varepsilon$ such that $0<\varepsilon<a_{\sigma} z_{\sigma}(t+1)$, and define $x=x(t)+\varepsilon e(\sigma), x^{\prime}=x(t+1)+\left((1-d) x_{\sigma}-x_{\sigma}(t+\right.$ $1)) e(\sigma)$, and $y=y(t)+\varepsilon e(\sigma)$. Then $e y=e y(t)+\varepsilon$ and $a\left(x^{\prime}-(1-d) x\right)=a(x(t+1)-(1-d) x(t))-a_{\sigma} z_{\sigma}(t+1)<$ $a z(t+1)-\varepsilon$. Thus $\left(x, x^{\prime}\right) \in \Omega$, and $y \in \Lambda\left(x, x^{\prime}\right)$, and from Theorem $6, w(b y(t))+p(t+1) x(t+1)-p(t) x(t) \geq$ $w\left(b y(t)+b_{\sigma} \varepsilon\right)+p(t+1) x^{\prime}-p(t) x$. This yields $w(b y(t)) \geq w\left(b y(t)+b_{\sigma} \varepsilon\right)$, a contradiction to the fact that $w$ is strictly increasing.

Proposition 15 For any $t \in \mathbb{N}, p_{\sigma}(t)>0$.

Proof: Suppose that there exists $t \in \mathbb{N}$ such that $p_{\sigma}(t)=0$. From Proposition 12, there exists a time-period $t_{i}>t$ such that $z_{\sigma}\left(t_{i}\right)>0$. From Proposition 14, this implies that $p_{\sigma}\left(t_{i}-1\right)>0$. If $t_{i}=t+1$, we obtain a contradiction. If $t_{i}>t+1$, we make as many (finite) appeals to Proposition 13 as is necessary to obtain a contradiction.

Proposition 16 For any $t \in \mathbb{N}$, and any $i \in 1, \cdots, n, x_{i}(t)>y_{i}(t) \Longrightarrow p_{i}(t+1)(1-d)=p_{i}(t)$.

Proof: Let $\varepsilon=x_{i}(t)-y_{i}(t)>0$, and define $x=x(t)-\varepsilon e_{i}, y=y(t)$, and $x^{\prime}=x(t+1)-(1-d) \varepsilon e(i)$. Then, it can be easily checked that $\left(x, x^{\prime}\right) \in \Omega$, and $y \in \Lambda\left(x, x^{\prime}\right)$. Using Theorem 6 , we obtain $(1-d) p_{i}(t+1) \varepsilon \geq p_{i}(t) \varepsilon$. We can now complete the proof of the claim by using Proposition 13.

Proposition $17 \liminf _{t \rightarrow \infty}\|p(t)\|<\infty$.

Proof: For any $t \in \mathbb{N}$, and any $i=1, \cdots, n$, define $x=x(t), z=z(t+1)+\left(e y(t) / a_{i}\right) e(i), x^{\prime}=z+(1-d) x, y=0$. Then $a z+e y=a z(t+1)+e y(t)$, and hence $\left(x, x^{\prime}\right) \in \Omega$ and $y \in \Lambda\left(x, x^{\prime}\right)$. We can now appeal to Theorem 6 to obtain $p_{i}(t+1)\left(e y(t) / a_{i}\right) \leq w(b y(t))-w(0) \leq w(b e)-w(0)$. Thus, there is $M>0$ such that for all $t \in \mathbb{N},\|p(t+1)\|(e y(t)) \leq M$. If $\liminf _{t \rightarrow \infty}\|p(t)\|=\infty$, then we must have $e y(t) \rightarrow 0$ as $t \rightarrow \infty$. But, then, the optimal program cannot be good, a contradiction to Proposition 10 that establishes the claim.

Proposition 18 For any $i \in 1, \cdots, n, z_{i}(t+1)>0 \Longrightarrow p_{i}(t+1) / p_{\sigma}(t+1) \geq a_{i} / a_{\sigma}$.

Proof: Suppose that for any $t \in \mathbb{N}$, and any $i=1, \cdots, n, z_{i}(t+1)>0$. Define $x=x(t), y=y(t), z=$ $z(t+1)-z_{i}(t+1) e(i)+\left(z_{i}(t+1)\left(a_{i} / a_{\sigma}\right)\right) e(\sigma), x^{\prime}=(1-d) x+z$. Since $a z+e y=a z(t+1)+e y(t) \leq 1,\left(x, x^{\prime}\right) \in \Omega$ and $y \in \Lambda\left(x, x^{\prime}\right)$. We can now appeal to Theorem 6 to obtain $p_{i}(t+1) z_{i}(t+1) \geq p_{\sigma}(t+1) z_{i}(t+1)\left(a_{i} / a_{\sigma}\right)$. Since $z_{i}(t+1)>0$, the proof of the claim is complete.

Proposition 19 Under Assumption 1, for any time-period $t \in \mathbb{N}$, and any $i \in 1, \cdots, n$,

$$
x_{i}(t)>0 \Longrightarrow(1-d)\left[\left(b_{i} / b_{\sigma}\right) p_{\sigma}(t+1)-p_{i}(t+1)\right] \leq\left[\left(b_{i} / b_{\sigma}\right) p_{\sigma}(t)-p_{i}(t)\right]
$$

Proof: We consider two cases: (i) $y_{i}(t)<x_{i}(t)$, and (ii) $y_{i}(t)=x_{i}(t)$.

Under case (i), let $\varepsilon=x_{i}(t)-y_{i}(t), x=x(t)+\varepsilon e(i), z=z(t+1), y=y(t)$ and $x^{\prime}=(1-d) x+z$. An appeal to Proposition 16 yields $(1-d) p_{i}(t+1)=p_{i}(t)$. Also, by Proposition 13, we have $(1-d) p_{\sigma}(t+1) \leq p_{\sigma}(t)$, and so

$$
\left(b_{i} / b_{\sigma}\right)\left[(1-d) p_{\sigma}(t+1)-p_{\sigma}(t)\right] \leq 0=\left[(1-d) p_{i}(t+1)-p_{i}(t)\right]
$$

Next, consider case (ii) where $y_{i}(t)=x_{i}(t)>0$. Let $0<\varepsilon<x_{i}(t), \nu=\left(b_{i} / b_{\sigma}\right) \varepsilon$, and note that from Assumption 1 that $\left(b_{i} / b_{\sigma}\right) \leq\left(a_{i} / a_{\sigma}\right) \leq 1$, which implies that $\nu \leq \varepsilon$. Define $x=x(t)-\varepsilon e(i)+\nu e(\sigma), y=$ 
$y(t)-\varepsilon e(i)+\nu e(\sigma), z=z(t+1), x^{\prime}=(1-d) x+z$, and note that $e y \leq e y(t), a z=a z(t+1)$ and $0 \leq y \leq x$. Thus, $\left(x, x^{\prime}\right) \in \Omega$, and $y \in \Lambda\left(x, x^{\prime}\right)$. Then from Theorem 6 , we obtain $p_{\sigma}(t+1)(1-d) \nu-p_{i}(t+1)(1-d) \varepsilon-p_{\sigma}(t) \nu+p_{i}(t) \varepsilon \leq$ 0 . This establishes the claim after transposing terms.

Proof of Theorem 7: Suppose that for some time-period $T$, and any $i=1, \cdots, n, z_{i}(T+1)>0$. Then by Proposition 18 and Assumption 1, we obtain

$$
p_{i}(T+1) \geq p_{\sigma}(T+1)\left(a_{i} / a_{\sigma}\right)>p_{\sigma}(T+1)\left(b_{i} / b_{\sigma}\right) .
$$

Also, we must have $x_{i}(t+1)>0$ for all $t \geq T$. Then, iterating on the result presented as Proposition 19, we obtain $p_{i}(t+1) \rightarrow \infty$ as $t \rightarrow \infty$, a contradiction to Proposition 17 . 
Atsumi, H. (1965). 'Neoclassical Growth and the Efficient Program of Capital Accumulation', Review of Economic Studies, vol. 32, pp. 127-136.

Bardhan, P. K. (1971). 'Optimum growth and allocation of foreign exchange', Econometrica, vol. 39, pp. 955-971.

Birner, J. (2002). The Cambridge Controversies in Capital Theory, London: Routledge.

Bliss, C. J. (1975). Capital Theory and the Distribution of Income, Amsterdam: North Holland.

Bliss, C. J. (1968). 'On putty-clay', Review of Economic Studies, vol. 35, pp. 105-122.

Brock, W. A. (1970). 'On existence of weakly maximal programmes in a multi-sector economy', Review of Economic Studies, vol. 37, pp. 275-280.

Bruno, M. (1967). 'Optimal accumulation in discrete capital models', Essay XI in K. Shell (ed.) Essays on the Theory of Optimal Economic Growth, Cambridge: MIT Press.

Cass, D. and Stiglitz, J. E. (1970). 'The implications of alternative savings and expectation hypotheses for choices of technique and patterns of growth', Journal of Political Economy, vol. 77, 586-627.

Dixit, A. (1990). 'Growth theory after thirty years', in P. Diamond (ed.) Growth, Productivity and Unemployment (Cambridge: The MIT Press).

Dobb, M. (1956). 'Second thoughts on capital-intensity of investment', Review of Economic Studies, vol. 24, pp. 33-42.

Dobb, M. (1960). An Essay on Economic Growth and Planning, New York: Monthly Review Press.

Dobb, M. (1961). Review of A. K. Sen's Choice of Techniques, Kyklos, vol. 14, 1961.

Dobb, M. (1967). 'The question of 'investment-priority for heavy industry', chapter 4 in Capitalism, Development and Planning, New York: International Publishers.

Dutta, P. and Mitra, T. (1989). 'Maximum theorems for convex structures with an application to the theory of optimal intertemporal allocation', Journal of Mathematical Economics, vol. 18, pp. 77-86.

Elaydi, S. N. (1999). An Introduction to Difference Equations, Berlin: Springer.

Gale, D. (1967). 'On optimal development in a multi-sector economy', Review of Economic Studies, vol. 34, pp. $1-18$.

Halevi, J. (1987) 'Investment planning', in (J. Eatwell, P. K. Newman and M. Milgate, eds.), The New Palgrave, London: MacMillan.

Khan, M. Ali (2000). 'Srinivasan on choice of technique again', Chapter 2 in Ranis-Raut (2000).

Khan, M. Ali and T. Mitra (2002). 'Optimal growth in the Robinson-Solow-Srinivasan model: the two-sector setting without discounting', mimeo, Cornell University.

Khan, M. Ali and T. Mitra (2003). 'Optimal growth under irreversible investment: a continuous time analysis', mimeo, Cornell University.

Koopmans, T. C. (1971). 'A model of a continuing state with scarce capital', in (G. Bruckmann and W. Weber, ed.), Contributions to the von Neumann Growth Model, New York: Springer-Verlag, pp. 11-22.

Koopmans, T. C. and Hansen, T. (1972). 'On the definition and computation of a capital stock invariant under optimization', Journal of Economic Theory, vol. 5, pp. 487-523.

McKenzie, L. W. (1968). 'Accumulation programs of maximum utility and the von Neumann facet', in (J. N. Wolfe, ed.), Value, Capital and Growth, Edinburgh: Edinburgh University Press, pp. 353-383.

McKenzie, L. W. (1986) "Optimal economic growth, turnpike theorems and comparative dynamics', in (K. J. Arrow and M. Intrilligator, eds.), Handbook of Mathematical Economics, Volume 3, New York: NorthHolland Publishing Company, pp. 1281-1355.

Mirrlees, J. A. (1962). 'Choice of techniques', Indian Economic Review vol. 6, pp. 93-102. 
Mitra, T. and Wan Jr., H. (1986). 'On the Faustmann solution to the forest management problem', Journal of Economic Theory vol. 40, pp. 229-249.

Naqvi, K. A. (1963). 'Machine tools and machines: a physical interpretation of marginal rate of saving', Indian Economic Review, vol. 7, 19-28.

Okishio, N. (1966). 'Technical choice under full employment in a socialist economy', Economic Journal, vol. 76, pp. 585-592.

Okishio, N. (1987). 'Choice of technique and the rate of profit', in (J. Eatwell, P. K. Newman and M. Milgate, eds.), The New Palgrave, London: MacMillan.

Raj, K. N. and Sen, A. K. (1961). 'Alternative patterns of growth under conditions of stagnant export earnings', Oxford Economic Papers, vol. 13, pp. 43-52.

Ramsey, F. (1928). 'A mathematical theory of savings', Economic Journal, vol. 38, pp. 543-559.

Ranis, G. and L. K. Raut (eds.) (2000). Trade, Growth and Development: Essays in Honor of Professor T. N. Srinivasan, Amsterdam: North Holland.

Robinson, J. (1960). Exercises in Economic Analysis, London: MacMillan.

Robinson, J. (1969). 'A model for accumulation proposed by J. E. Stiglitz', Economic Journal, vol. 79, pp. 412-413.

Rudin, W. (1976). Principle of Mathematical Analysis, New York: McGraw Hill.

Sen, A. K. (1960). Choice of Techniques, Oxford: Basil Blackwell.

Sen, A. K. (1968). 'Optimal savings, technical choice and the shadow price of labour', introduction to the third edition of Sen (1960).

Solow, R. M. (1962a). 'Some problems in the theory and practice of economic planning", Economic Development and Cultural Change, vol. 10, pp. 216-222.

Solow, R. M. (1962b). 'Substitution and fixed proportions in the theory of capital', Review of Economic Studies, vol. 29, pp. 207-218.

Solow, R. M. (2000) 'Srinivasan on choice of technique', Chapter 1 in Ranis-Raut (2000).

Srinivasan, T. N. (1962a). 'Investment criteria and choice of techniques of production', Yale Economic Essays, vol. 1, pp. 58-115.

Srinivasan, T. N. (1962b). Review of Sen (1960) in Journal of Political Economy, vol. 70, pp. 306-307.

Stiglitz, J. E. (1968). 'A note on technical choice under full employment in a socialist Economy', Economic Journal, vol. 78, pp. 603-609.

Stiglitz, J. E. (1970). 'Reply to Mrs. Robinson on the choice of technique', Economic Journal, vol. 80, pp. 420-422.

Stiglitz, J. E. (1973). 'Recurrence of techniques in a dynamic economy', in J. Mirrlees and N. H. Stern (eds.) Models of Economic Growth, New York: John-Wiley and Sons.

Stiglitz, J. E. (1990). 'Comments: some retrospective views on growth theory', in P. Diamond (ed.) Growth, Productivity and Unemployment (Cambridge: The MIT Press).

von Weizsäcker, C. C. (1965). 'Existence of optimal programs of accumulation for an infinite time horizon', Review of Economic Studies, vol. 32, pp. 85-104.

von Neumann, J. (1935-36). 'Über ein Ökonomisches Gleichungs-System und eine Verallgemeinerung des Brouwerschen Fixpunktsatzes', in K. Menger (ed.) Ergebnisse eines Mathematischen Kolloquiums, No. 8. Translated as "A model of general economic equilibrium", Review of Economic Studies, vol. XIII, pp. 1-9. 